Revue des patrimoines

Le patrimoine religieuX des $\mathrm{XIX} \mathrm{X}^{\mathrm{e}}$ et $\mathrm{XX} \mathrm{X}^{\mathrm{e}}$ siècles

\title{
La sélection des objets à protéger dans les églises de la Reconstruction : l'exemple de la Manche
} (1944-1974)

\section{Élisabeth Marie}

\section{(2) OpenEdition}

\section{Journals}

Édition électronique

URL : http://journals.openedition.org/insitu/6069

DOI : 10.4000/insitu.6069

ISSN : 1630-7305

Éditeur

Ministère de la culture

Référence électronique

Élisabeth Marie, «La sélection des objets à protéger dans les églises de la Reconstruction : l'exemple de la Manche (1944-1974)», In Situ [En ligne], 11 | 2009, mis en ligne le 18 avril 2012, consulté le 19 avril 2019. URL : http://journals.openedition.org/insitu/6069 ; DOI : 10.4000/insitu.6069

Ce document a été généré automatiquement le 19 avril 2019

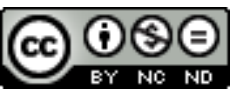

In Situ Revues des patrimoines est mis à disposition selon les termes de la licence Creative Commons Attribution - Pas d'Utilisation Commerciale - Pas de Modification 4.0 International. 


\title{
La sélection des objets à protéger dans les églises de la Reconstruction : l'exemple de la Manche (1944-1974)
}

\author{
Élisabeth Marie
}

\section{Introduction}

1 Dans la Manche, sur trois cents églises ou chapelles concernées par les destructions de 1944 (sur un total d'environ sept cents), un peu plus de la moitié des édifices était considérée comme complètement détruits ou gravement endommagés, le reste étant utilisable en partie ou légèrement atteint ${ }^{1}$. Certains cantons ont ainsi perdu la majeure partie de leurs édifices religieux (dix doyennés ont un taux de destruction de cinquante pour cent) $)^{2}$. 


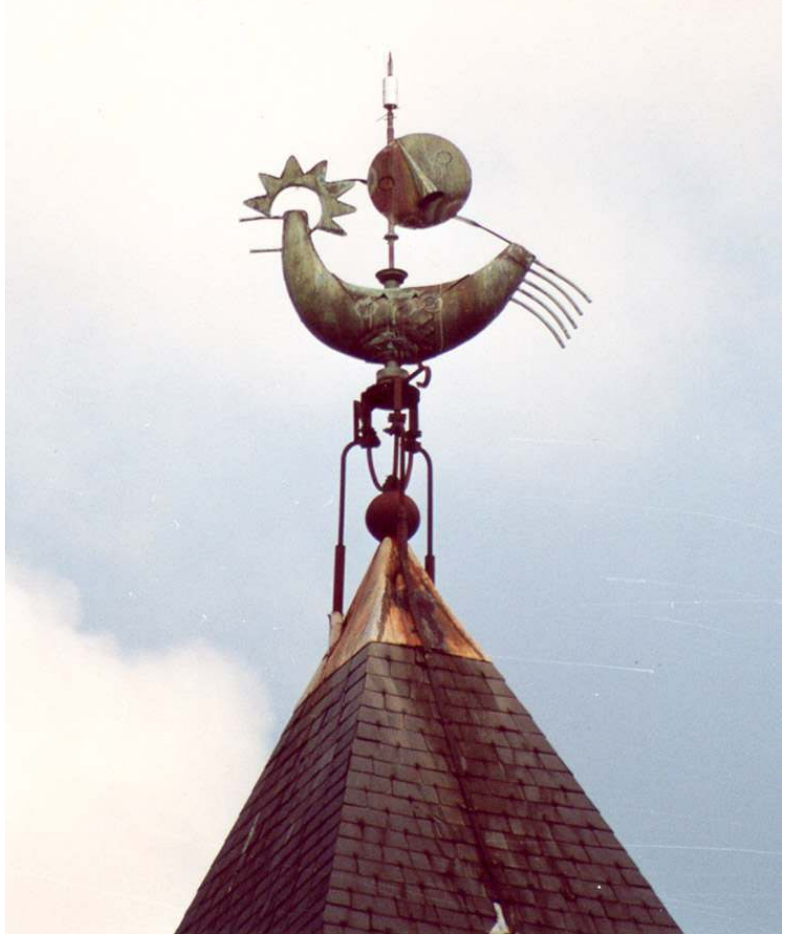

Coq du clocher de l'église Saint-Jean-Baptiste d'Agneaux (Manche), par Jean Lambert-Rucki, 1954

(c) Conseil général de la Manche/CAOA

L'ampleur des destructions du patrimoine religieux dans la Manche a eu pour corollaire un travail de restauration et de création important entre 1944 et 1974 . De nombreux artistes sont venus œuvrer à « la Renaissance des clochers » dans le département sinistré. Parallèlement à l'étude menée sur les édifices par le service des Monuments historiques, à l'échelle de la Basse-Normandie ${ }^{3}$, un inventaire thématique du mobilier a été entrepris pour la Manche, par la Conservation départementale des objets d'art. Pour faire suite à ces enquêtes, quarante objets ont été inscrits au titre des monuments historiques, tant sculptures, que tentures, mobilier liturgique, peintures ou objets d'orfèvrerie (fig. $\mathbf{n}^{\circ} \mathbf{1}$ ).

\section{Les objectifs d'une première sélection}

\section{La conservation matérielle}

Lors des opérations de récolement, nous avions pu repérer un certain nombre d'objets de qualité datant de cette période et identifier des artistes importants pour l'histoire de l'art. Mais nous avons également été alertés par le mauvais état de conservation des œuvres. Bien qu'encore assez récent, ce patrimoine se dégrade en effet très vite, spécialement les objets les plus fragiles, comme les tapisseries ou les peintures. Plusieurs facteurs expliquent ces dégradations : le manque d'entretien d'une part, en raison du désintérêt de la population pour les églises de la période (dites «modernes» avec une connotation négative) et du problème de la désertification des églises en milieu rural, les matériaux 
employés d'autre part, (matériaux dits " pauvres » comme le tilleul pour la sculpture ou les techniques mixtes) et enfin, l'environnement souvent dégradé du bâtiment lui même...

Figure 2

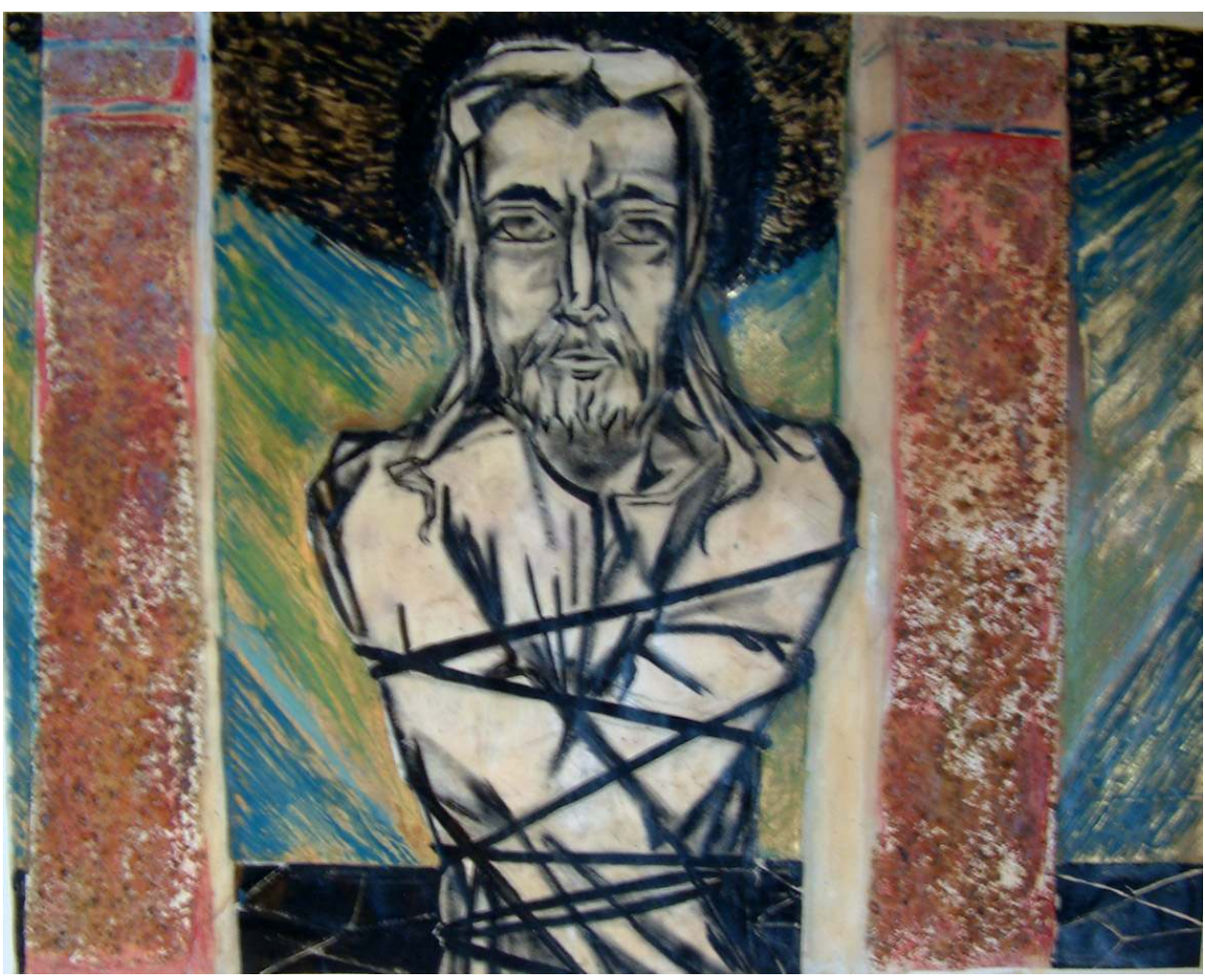

Première station du chemin de croix de Quibou par Helen Mai, maquette

(c) Conseil général de la Manche/CAOA 
Figure 3

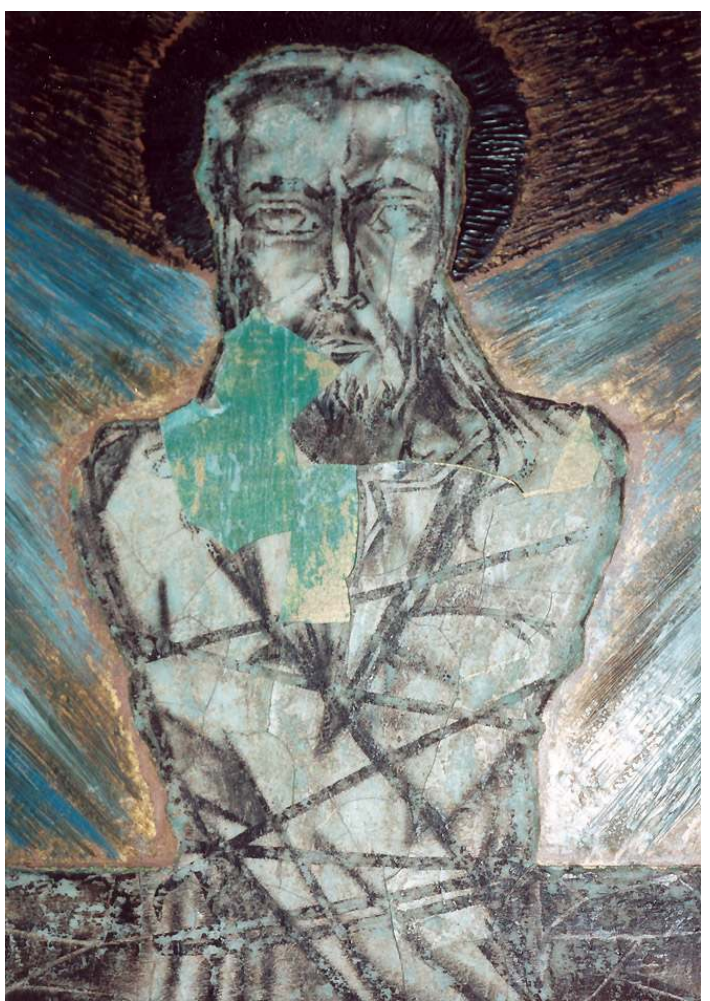

Station du chemin de croix de Quibou par Helen Mai, détail montrant le déplaquage de la couche picturale

(c) Conseil général de la Manche/CAOA

4 Le chemin de croix de Quibou (1955), réalisé par Helen Mai (pseudonyme de Jeanne Lartigue) en technique mixte (peinture à l'huile et peinture à la cire à chaud dite «à l'encaustique » sur bois, associant retouches à la paraffine à froid et granité de sable polychrome, papier, etc.) présente des détériorations importantes aggravées par le climat lumineux du chœur largement vitré (fig. $\mathbf{n}^{\circ} 2, \mathbf{n}^{\circ} 3$ ). À l'église de Graignes, l'humidité excessive, due au mauvais état des réseaux de béton, a entraîné des déformations sur la tapisserie de François Chapuis (1928- ) (fig. $n^{\circ} 4, n^{\circ}$ 5), également décolorée du fait d'un fort taux de luminosité (dalles de verre). 
Figure 4

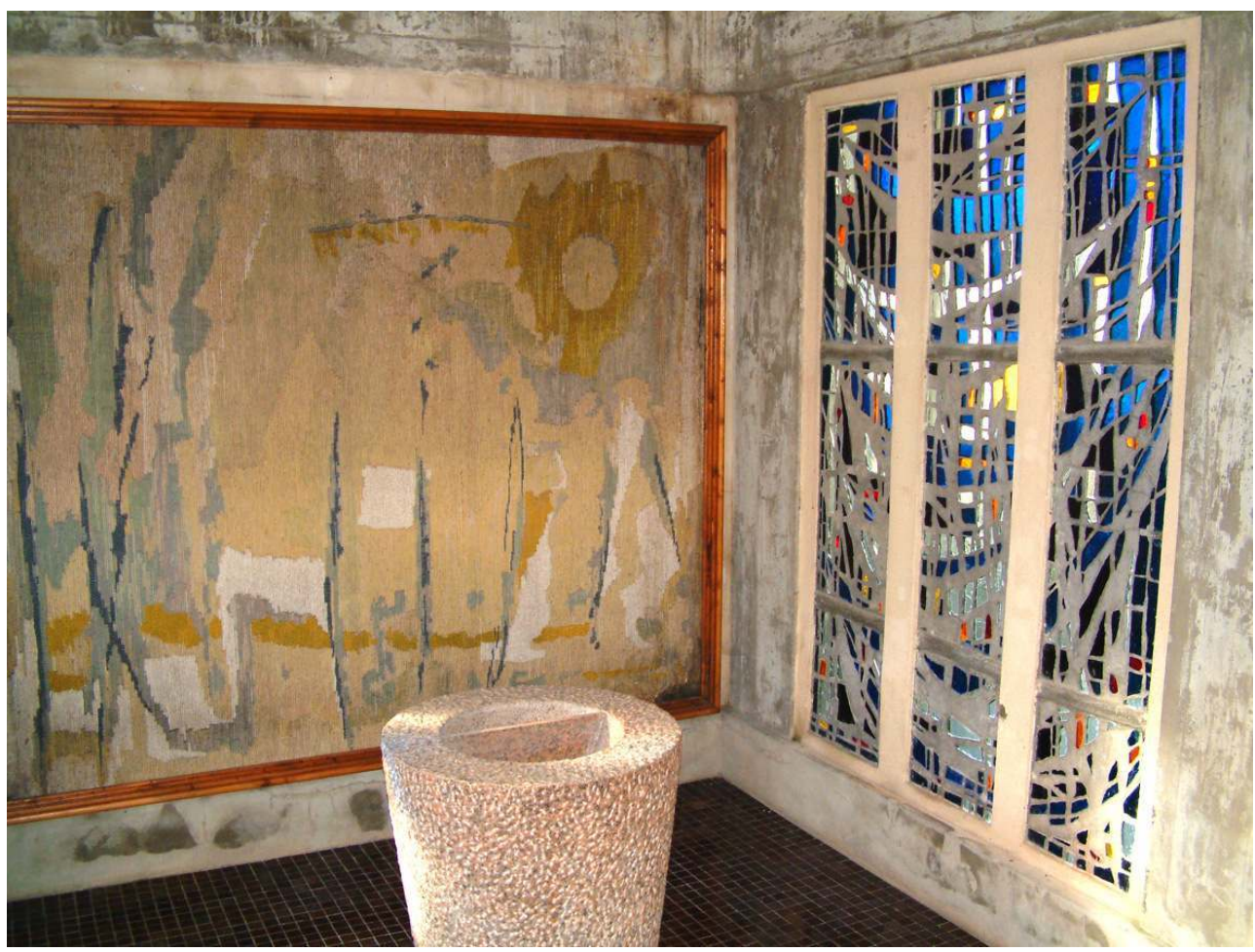

Tapisserie de François Chapuis, dans la chapelle des fonts baptismaux de Graignes, avant restauration

(c) Conseil général de la Manche/CAOA 


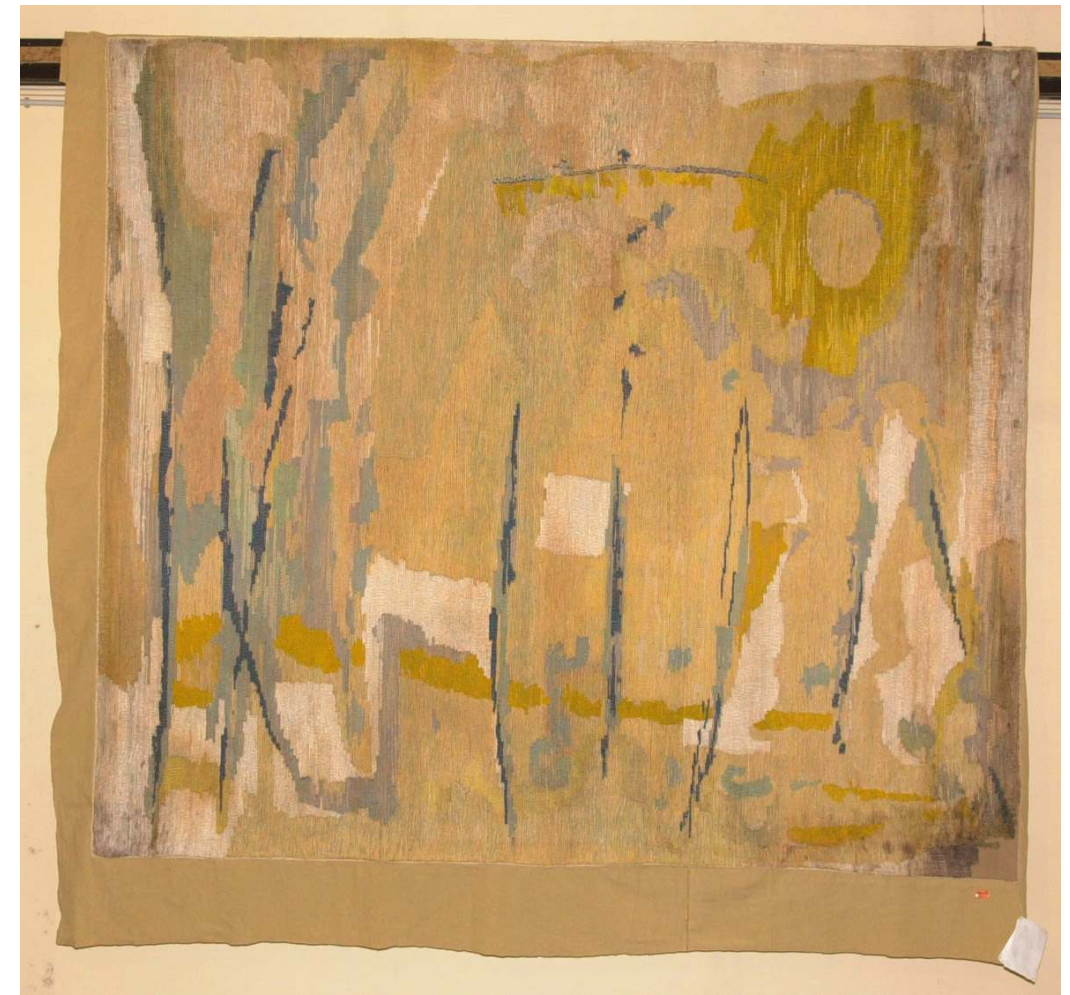

Tapisserie de François Chapuis de Graignes, après restauration

(C) Conseil général de la Manche/CAOA

\section{La connaissance}

5 Il paraissait aussi important de commencer à rassembler la documentation sur ces œuvres, sources déjà menacées: en recueillant les témoignages des acteurs de cette époque (artistes, architectes, artisans, entreprises, prêtres...), mais aussi, en dressant un état des lieux des archives existantes (avec le concours des diverses structures concernées) et des fonds d'ateliers concernés (en rapport avec des artistes vivants ou leurs familles). Les sources photographiques offrent aussi une documentation technique intéressante, comme, par exemple, une série de photographies faites au moment de la fonte, réalisée in situ, des portes en bronze de l'église Notre-Dame de Saint-Lô, « la porte des hommes » et « la porte de Dieu », par le sculpteur Jean Bernard (1908-1994) en 1972 (fig. $n^{\circ} 6, n^{\circ} 7$ ). 
Figure 6

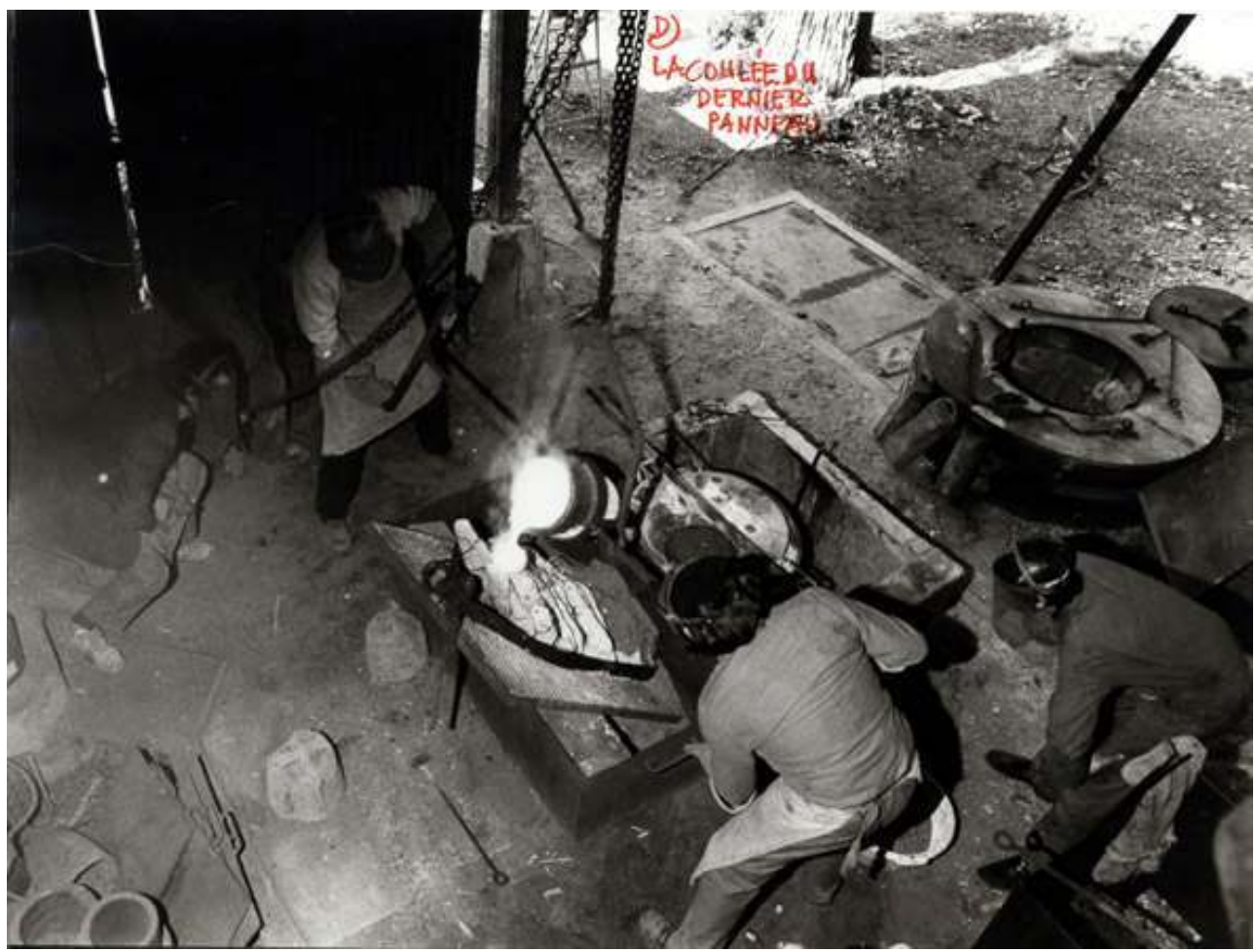

Fonte du bronze pour la réalisation des portes de Notre-Dame de Saint-Lô, coulée du dernier panneau (c) Conseil général de la Manche/CAOA 
Figure 7

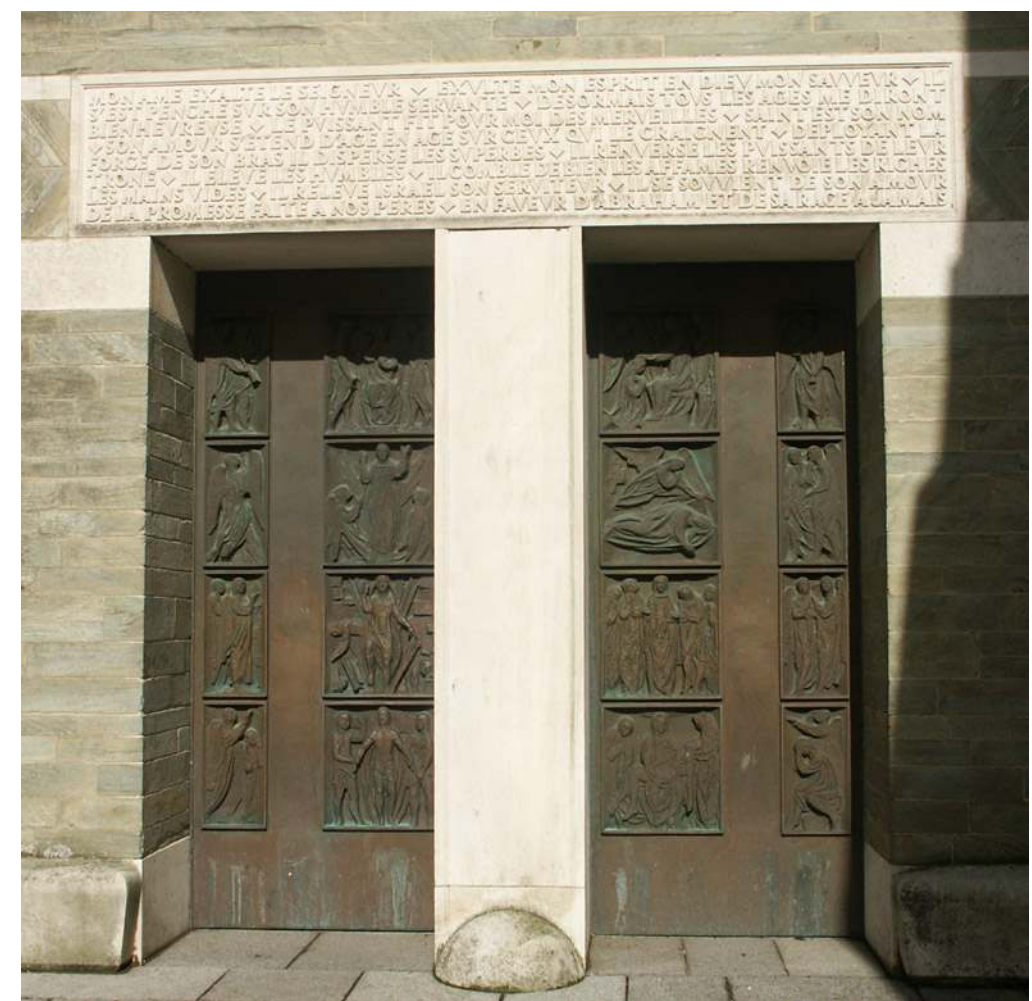

Portes en bronze de Jean Bernard, à l'église Notre-Dame de Saint-Lô

(c) Conseil général de la Manche/CAOA

\section{La protection, la diffusion et la mise en valeur}

6 La protection au titre des monuments historiques en 2003 et 2004 a permis d'engager des opérations de restauration et d'initier un intérêt patrimonial, en lien avec les commémorations du soixantenaire, en 2004. Cette phase devait aboutir à une publication courte et en même temps suffisamment représentative des différents aspects de ce patrimoine (plaquettes conçues pour accompagner des circuits de visite) ${ }^{4}$.

7 Ces objectifs établis par la conservation ont en fait contribué à définir les premiers éléments d'une sélection.

\section{De l'inventaire au choix des œuvres}


Figure 8

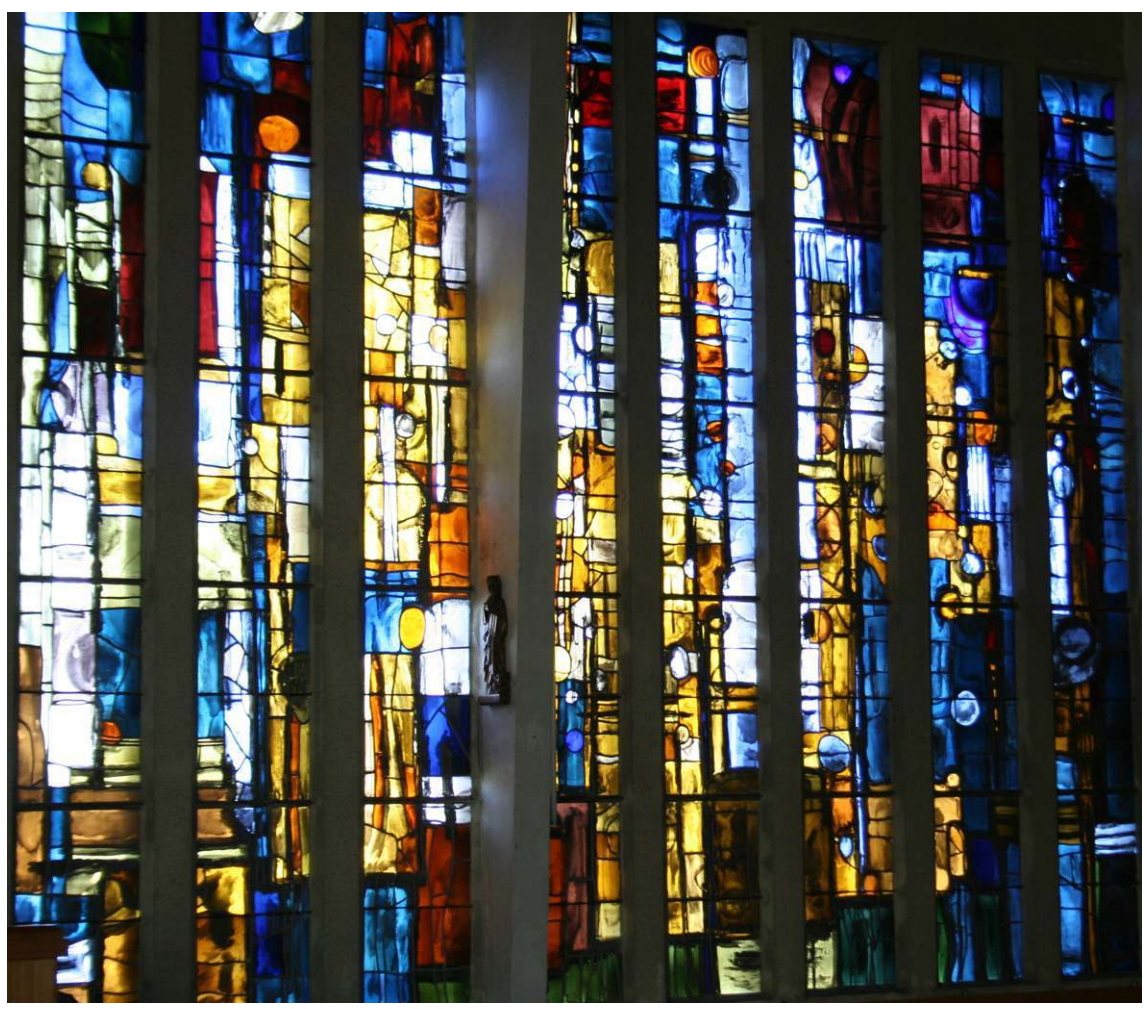

Vitrail de François Chapuis à l'église de Saint-Jean-des-Baisants (édifice ISMH en raison de l'intérêt des verrières)

(c) Conseil général de la Manche/CAOA

8 Une enquête réalisée en 1944 a servi de base à notre étude. Elle concerne les destructions des édifices dans la Manche et dresse un bilan des églises reconstruites (établi dans les années 60) faisant état des reconstitutions mobilières avec la liste des objets et du décor par édifice et parfois, les dates et auteurs. Bien que ce bilan, arrêté avant la fin réelle du renouvellement mobilier, au milieu des années soixante-dix, se soit révélé incomplet et parfois inexact, il nous a permis de constituer une première liste de travail. Sur quelque cent soixante églises répertoriées pour l'étude, parce que conservant un objet ou un ensemble réalisé dans l'immédiat après-guerre - soit environ cent trente objets ou ensembles, auxquels s'ajoutent les vitraux et dalles de verre, nous avons sélectionné, dans un premier temps, une trentaine d'édifices pour un inventaire proprement dit comprenant archives, terrain et restitution documentée. La deuxième phase consiste en un inventaire exhaustif, notamment du corpus de vitraux ${ }^{5}$ (fig. $\mathbf{n}^{\circ} \mathbf{8}$ ).

\section{La sélection par rapport à un contexte administratif et architectural}

9 Les édifices sélectionnés sont représentatifs des différentes catégories de reconstructions (restauration "monument historique», reconstruction partielle, style régionaliste, tendance moderniste). Ces catégories recouvrent des structures de financement et des choix en matière de programme très distincts ; elles déterminent le mode de financement et de suivi administratif des édifices reconstruits et induisent la localisation des sources d'archives qui les concernent. 


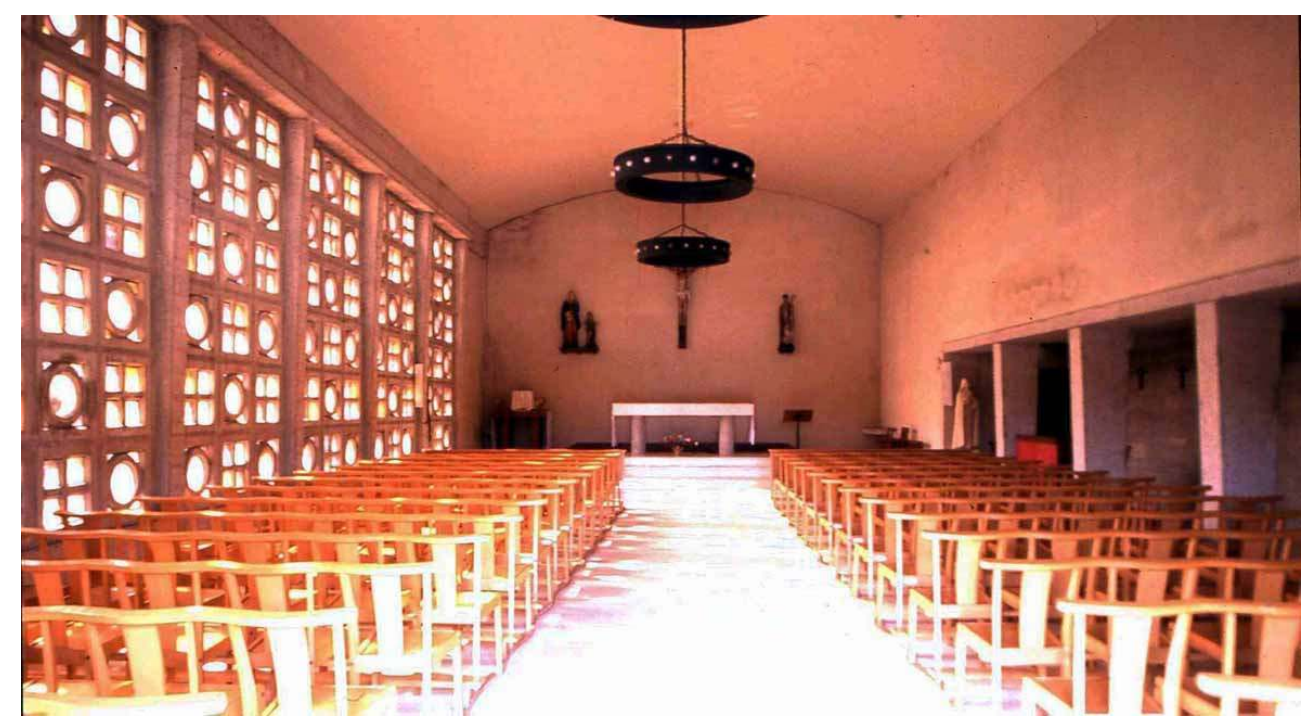

Église de Laulne

Phot. Alain Nafilyan. (c) Conseil général de la Manche/CAOA

10 La sélection d'objets en rapport avec ces catégories montre comment les choix en matière de programmes architecturaux, liés au contexte administratif, ont pu influencer la création de mobilier ; elles conditionnent dans une certaine mesure, en effet, les budgets alloués à la décoration intérieure et l'acquisition d'objets, mais aussi l'implication des architectes concernant la partie décorative et mobilière et surtout le choix des créateurs.

D’une manière générale, lorsque la plus grande part du financement est consacrée au bâtiment, le mobilier Reconstruction est pauvre. Ainsi, dans les réalisations architecturales d'esprit moderne, comme à Laulne (fig. $\mathbf{n}^{\circ}$ 9) ou dans les grandes églises de la région des marais, dans le centre Manche, pourtant de style régionaliste - donc plutôt favorable à une tendance décorative, il n'y a que très peu de mobilier datant des années cinquante (église Saint-Sébastien du Plessis). (fig. $\mathbf{n}^{\circ}$ 10) 
Figure 10

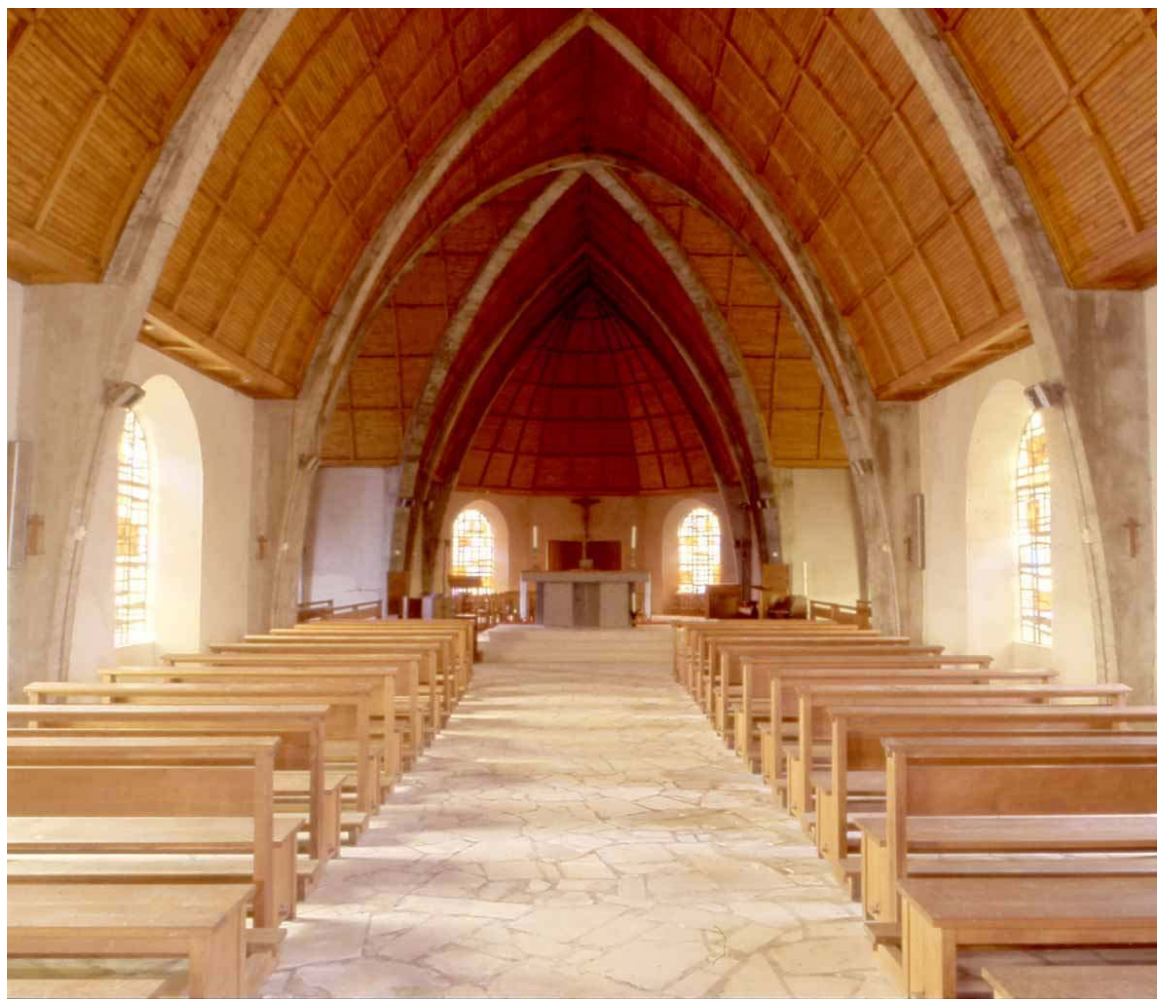

Église Saint-Sébastien du Plessis (Le Plessis-Lastelle)

Phot. Alain Nafilyan. (c) Conseil général de la Manche/CAOA

\section{Figure 11}

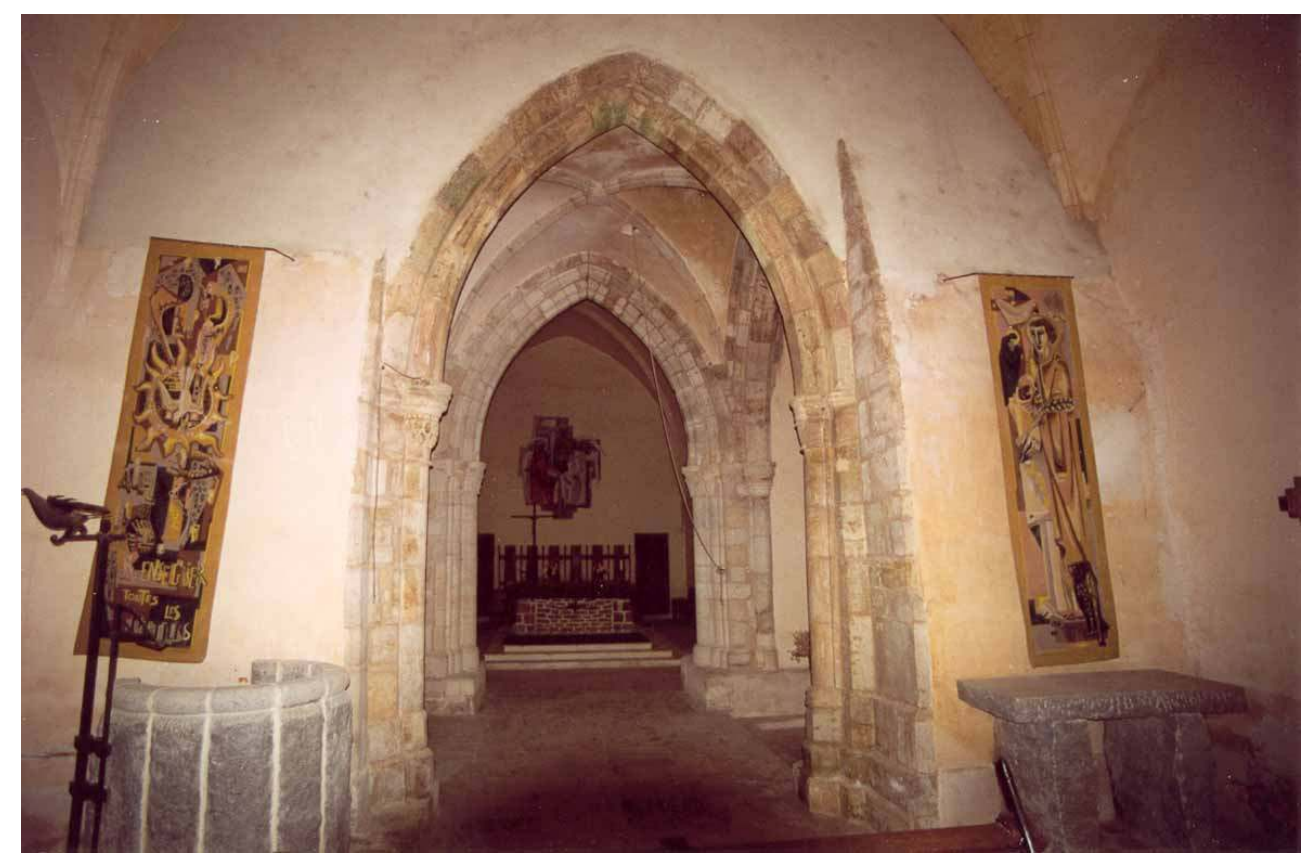

Église Saint-Jean-Baptiste de Lastelle (le Plessis-Lastelle) (c) Conseil général de la Manche/CAOA 
12 En revanche, lorsqu'une église est simplement restaurée, même dans un style contemporain, le mobilier est de fait plus présent; il est dû surtout à de grands noms et très peu à des artistes locaux (église d'Agneaux). A fortiori, s'il s'agit d'une église légèrement atteinte, la reconstitution mobilière est préservée et l'implication du clergé et de la commission diocésaine d'art sacré est plus importante. Cette dernière propose des artistes et conseille le clergé (notamment en matière de programme iconographique et d'aménagement liturgique: place de l'autel, du tabernacle...). Les archives conservées seront aussi plus abondantes et plus riches. Le décor de l'église Saint-Jean-Bastiste de Lastelle s'est ainsi enrichi de tapisseries de François Chapuis, d'une peinture murale de Maurice Rocher (1918-1995), de vitraux de François Chapuis et J. Ghiglione, d'un Christ en bronze, de mobilier liturgique dessiné par l'architecte Georges Pouleaud (fig. $\mathbf{n}^{\circ} \mathbf{1 1}$ ).

Figure 12

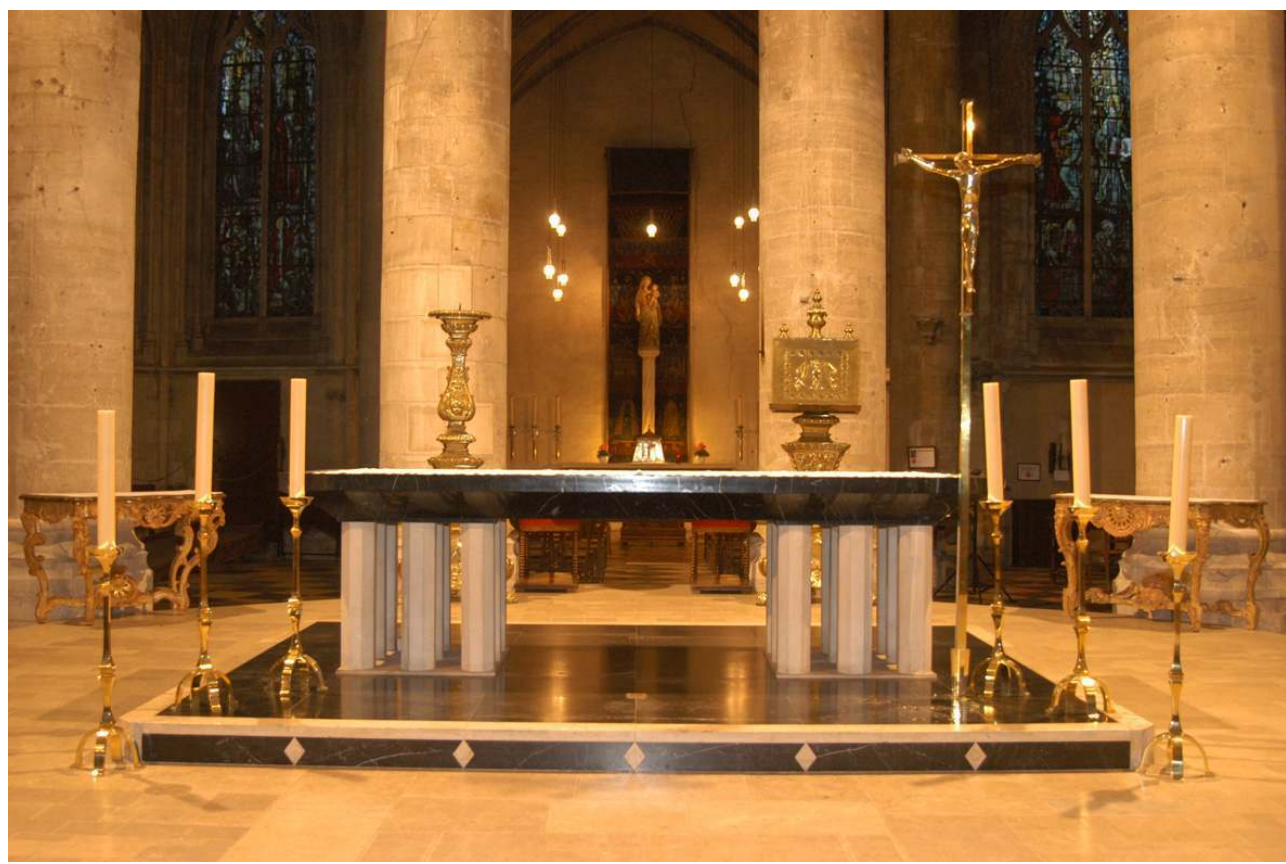

Chœur de l'église Notre-Dame de Saint-Lô : mobilier liturgique dessiné par Y-M. Froidevaux et réalisé par la maison Chéret, Christ de Jean Dambrin

Phot. Pierre-Yves Le Meur. (c) Conseil général de la Manche/CAOA

Lorsqu'il s'agit d'une restauration "monument historique " (comme à l'abbatiale de Lessay, à l'église Notre-Dame de Saint-Lô (fig. $\mathbf{n}^{\circ} \mathbf{1 2}$ ) ou à l'église Saint-Malo de Valognes), l'architecte en chef (Yves-Marie Froidevaux [1907-1983], pour la Manche) est très impliqué en ce qui concerne le mobilier, qui est également très présent, et ce sont souvent les mêmes artistes à qui l'architecte fait appel: Simone Flandrin-Latron, Max Ingrand (1908-1969), Jean-Paul Froidevaux, Lucien Jeay, Jean Dambrin, etc.

Enfin, certaines églises peu atteintes par les bombardements ont connu des pillages. Bien que non reconstruites, elles ont bénéficié d'indemnités pour le renouvellement de leur mobilier cultuel. C'est le cas par exemple de l'église de La Haye-du-Puits.

Il faut donc tenir compte de ces contextes dans la sélection patrimoniale des objets car cette dernière en portera nécessairement témoignage. 


\section{Aspects empiriques et plus aléatoires de la sélection}

Figure 13

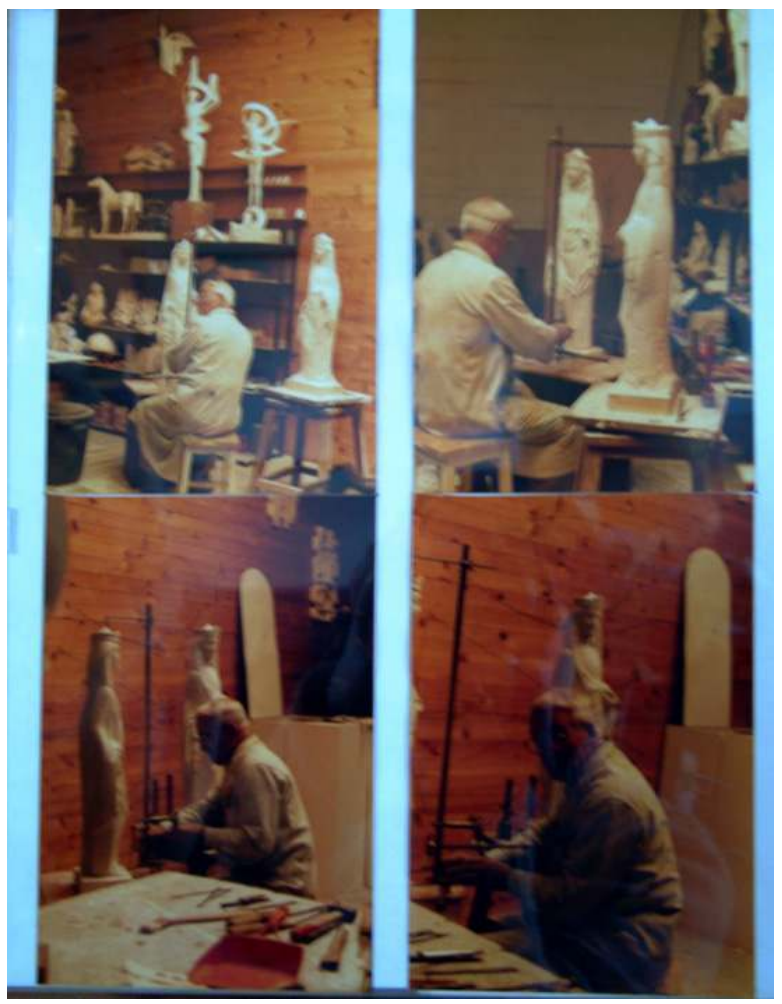

Atelier d'Étienne Rebuffet

(C) Conseil général de la Manche/CAOA

16 Certains artistes ou certains ensembles mobiliers bénéficient d'une recherche plus approfondie que d'autres en fonction de l'existence de fonds d'ateliers, de sources d'archives importantes, des témoins et acteurs des chantiers (témoignages de prêtres, d'entrepreneurs)... Cette meilleure connaissance permet aussi une sélection plus représentative. C'est le cas, par exemple, de l'œuvre d'Étienne Rebuffet. Le sculpteur, venu dans la Manche pour la Reconstruction s'est définitivement fixé à Agneaux. Son atelier conserve encore une importante documentation sur ses œuvres; l'exploitation de cette source particulière aide à l'exhaustivité de l'inventaire et à la pertinence de la sélection (fig. $\mathbf{n}^{\circ}$ 13) (voir fig. $\mathbf{n}^{\circ} 27, \mathbf{n}^{\circ} 28$ ).

\section{Les critères de protection au titre des monuments historiques}

La commission de protection est le lieu d'aboutissement du processus de sélection.

Lors des Commissions départementales des objets mobiliers, dans les présentations, les objets proposés à l'inscription étaient regroupés par catégorie (sculpture, textile, mobilier liturgique, orfèvrerie, chemins de croix) puis par auteurs. Trois ensembles d'objets constituant le décor d'un édifice ont été présentés à part. Les objets proposés à l'inscription étaient associés, lors des présentations, à d'autres œuvres comparables, 
inventoriés mais non sélectionnés, de manière à justifier les sélections ou, le cas échéant, à modifier les choix. De même, certains objets ont été simplement montrés aux membres afin de permettre une évaluation plus juste de la valeur de chaque objet, au sein du corpus existant.

Les deux commissions consacrées à cette thématique ont permis de débattre, d'affiner et de préciser les critères généraux de protection au titre des monuments historiques. Ont donc été retenus plus précisément pour ce qui concerne ce patrimoine particulier :

1. la qualité intrinsèque de l'œuvre : facture, qualité du matériau employé, qualité d'exécution, intérêt iconographique ;

2. la capacité de l'œuvre à s'inscrire dans un courant artistique - celui des années cinquante -, qui est à la fois le courant de «la modernité », à situer dans la lignée de l'École de Paris, mais aussi celui du renouveau des arts sacrés;

3. le caractère représentatif de l'œuvre d'un artiste ou son originalité ;

4. la personnalité de l'artiste (à savoir s'il s'agit d'un artiste important pour la Manche, ayant un ancrage dans le département, ou bien d'un artiste de renommée nationale);

5. le rapport avec l'édifice dans lequel l'œuvre s'inscrit, son appartenance à un ensemble cohérent indissociable de l'œuvre architecturale ;

6. l'intérêt documentaire: un intérêt dans la mesure où l'œuvre participe à augmenter la connaissance d'un artiste ou d'un groupe d'artistes, du fonctionnement des instances de la Reconstruction, de la présence et de la mentalité des commanditaires, ou encore des courants liturgiques novateurs (place et forme du tabernacle par exemple)...

Ainsi, quarante objets ont été inscrits au titre des monuments historiques, représentatifs des diverses catégories d'objets, des différents critères retenus et du corpus d'édifices étudiés (dix-sept églises sont concernées par les protections; vingt-deux artistes ou exécutants sont représentés).

21 Le décor de l'église d'Agneaux (fig. $\mathbf{n}^{\circ}$ 14) a été inscrit au titre des monuments historiques dans sa globalité (chaque objet possédant son propre arrêté), en raison de l'intérêt intrinsèque des œuvres mais aussi et surtout, du lien historique et artistique qui préside à sa création. La protection concerne tout le décor, excepté les vitraux qui ne pouvaient être protégés en tant qu'objets : chemin de croix en pierre sculptée, cinq sculptures, deux tabernacles, le couvercle des fonts baptismaux, le coq du clocher. C'est une église qui a été partiellement reconstruite, réaménagée et décorée entre 1951 et 1956. À son inauguration, l'église fait l'unanimité, cas assez rare. L'architecte Jacques Prioleau (1913- ) s'implique dans la réalisation du décor en choisissant de s'entourer d'un groupe d'artistes de renom, liés à l'École de Paris, ou soutenus par la revue L'Art sacré: Léon Zack (1892-1980) et Maxime Adam-Tessier (1920-2000) qui collaborent à la réalisation des deux tabernacles (fig. $\mathbf{n}^{\circ}$ 15) et du chemin de croix en 1953 et 1956 (voir fig. n 33). 
Figure 14

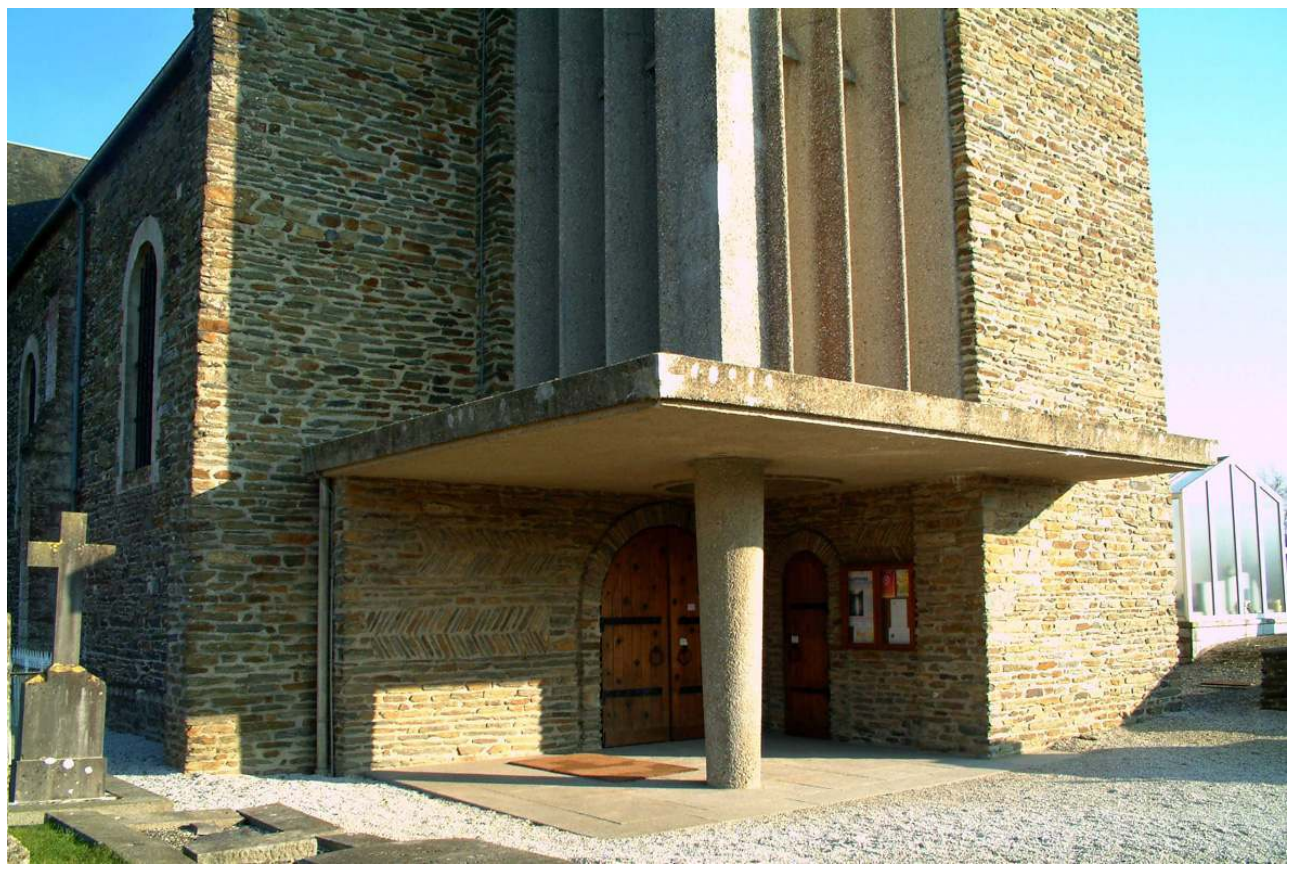

Église d'Agneaux, porche

(c) Conseil général de la Manche/CAOA

Figure 15

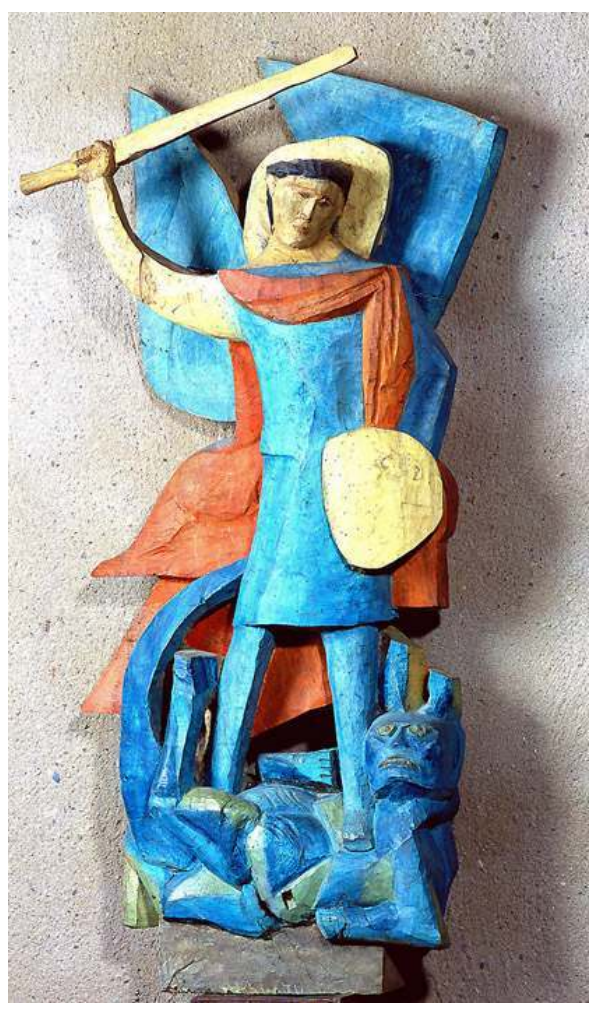

Saint Michel terrassant le dragon, haut-relief de Philippe Kaëppelin à l'église d'Agneaux Phot. Pierre-Yves Le Meur. (c) Conseil général de la Manche/CAOA 
22 À Jean Lambert-Rucki (1888-1967), on doit le coq du clocher (voir fig. $\left.\mathbf{n}^{\circ} \mathbf{1}\right)$ et le couvercle des fonts baptismaux (fig. $\mathbf{n}^{\circ}$ 16). Philippe Kaëppelin (1918- ) réalise cinq sculptures (fig. $\mathbf{n}^{\circ}$ 17). Simone Flandrin-Latron enfin, dessine les maquettes des vitraux. Cuvre d'un artiste vivant au moment de la protection, l'édifice a simplement reçu le label Patrimoine $X X^{e}$.

Figure 16

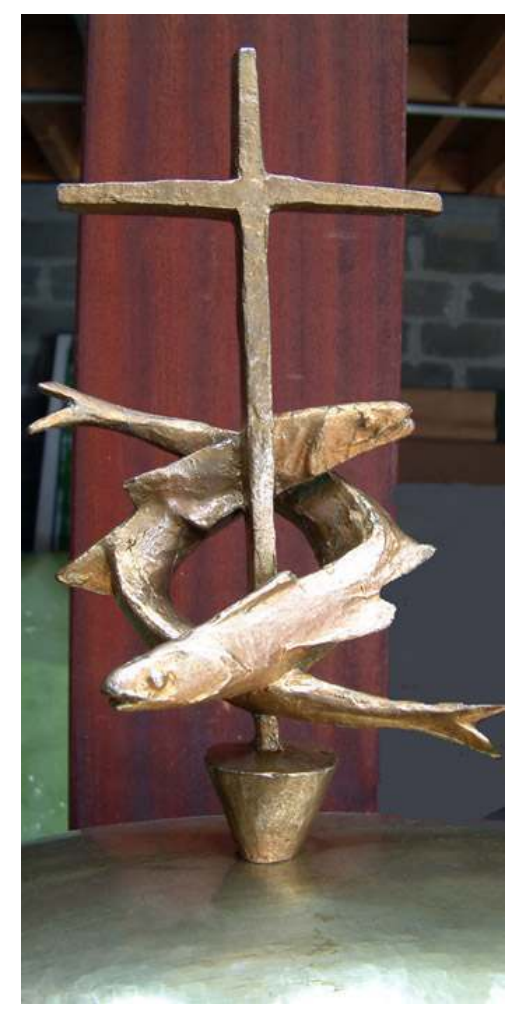

Couronnement des fonts baptismaux par Lambert-Rucki, église d'Agneaux (c) Conseil général de la Manche/CAOA 
Figure 17

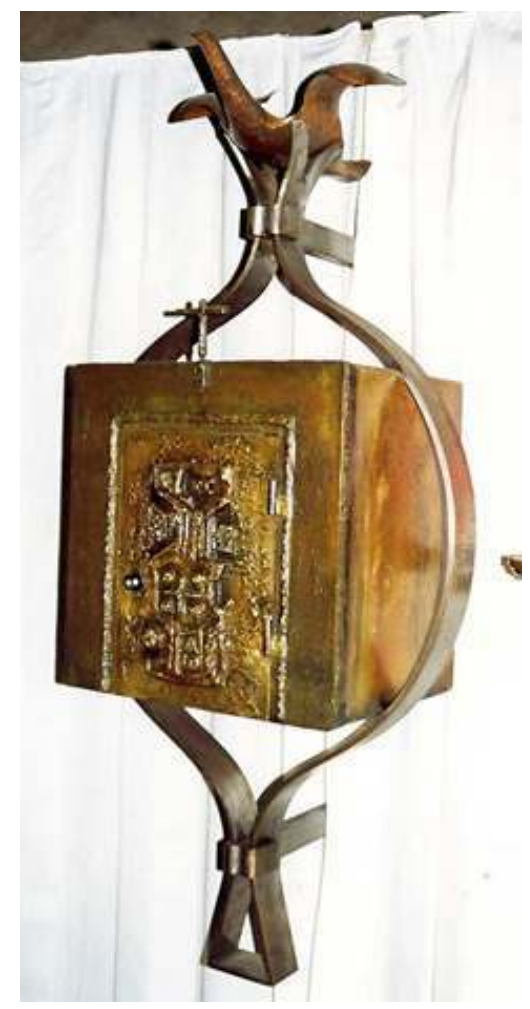

Tabernacle de Léon Zack (maquette) et Maxime Adam-Tessier (réalisation), à l'église d'Agneaux (C) Conseil général de la Manche/CAOA

23 Parmi les onze sculptures (dont un bas-relief compris dans la protection de l'édifice), le Christ en croix de Jean Lambert-Rucki vers 1958 à Lessay (fig. $\mathbf{n}^{\circ}$ 18), œuvre originale de l'artiste (à côté des nombreuses éditions de la maison Chéret) a été choisi pour sa qualité artistique et son caractère représentatif de l'œuvre du sculpteur, tournée vers les sources religieuses de l'art africain. Un autre Christ en croix, à La Chapelle-Enjuger, de Philippe Kaëppelin (fig. $\left.\mathbf{n}^{\circ} 19\right)$ a été sélectionné pour son intérêt iconographique. L'artiste présente un Christ figuré dans la gloire de sa Résurrection, triomphant de la mort. 
Figure 18

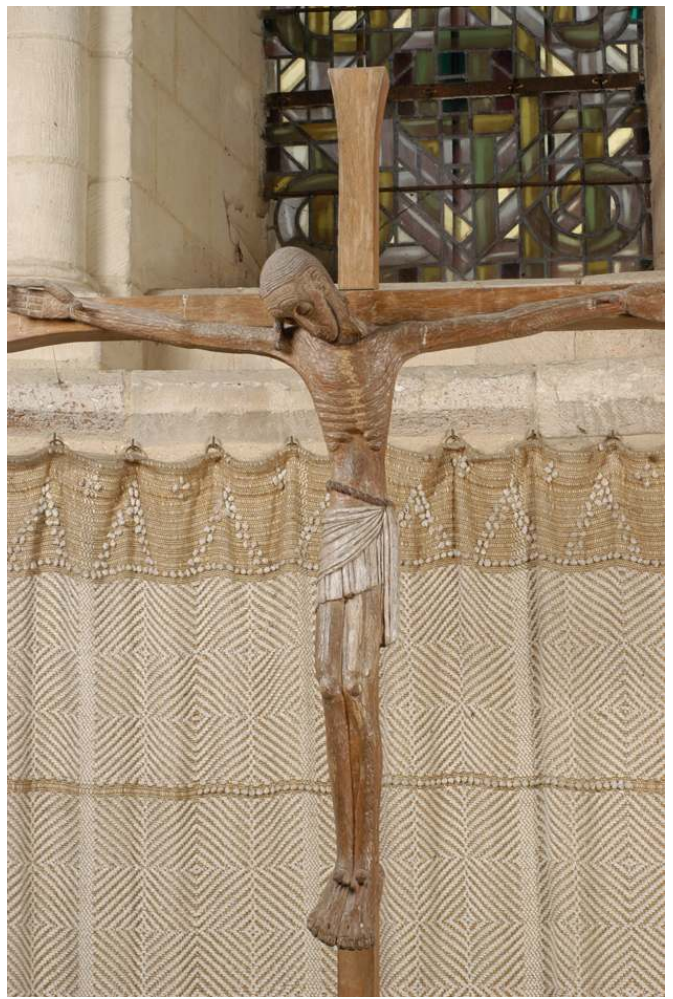

Christ en croix de Jean Lambert-Rucki, à l'abbatiale de Lessay

Phot. Pierre-Yves Le Meur. (C) Conseil général de la Manche/CAOA 
Figure 19

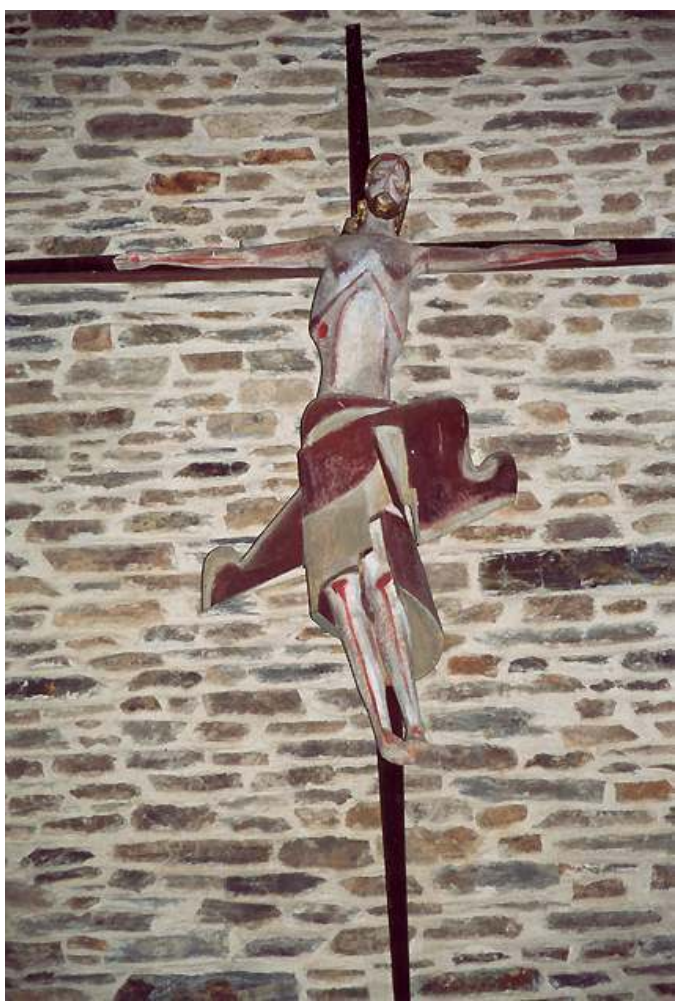

Christ en croix de Philippe Kaëppelin, église de La Chapelle-Enjuger

(c) Conseil général de la Manche/CAOA

Dix tentures (dont une paire et une série de quatre pièces) ont été protégées, ce qui correspond à la quasi-totalité des tentures repérées, en raison, d'une part de la qualité des maquettes et des exécutions des pièces textiles, de la relative rareté de ces objets, d'autre part, et enfin, de leur fragilité. Objets rares dans l'œuvre de l'artiste, les tapisseries de François Chapuis au Plessis-Lastelle (exécution manufacture d'Aubusson) (voir fig. $\mathbf{n}^{\circ}$ 11), à Graignes (voir fig. $\mathbf{n}^{\circ}$ 5) et au Mesnil-Véneron (exécution Jacques Plasse-Lecaisne) (fig. $\mathbf{n}^{\circ}$ 20); la tapisserie de Simone Flandrin-Latron (carton) et Charles Giraud (exécution) à Notre-Dame de Saint-Lô (fig. $\mathbf{n}^{\circ}$ 21); la tenture de Helen Mai (exécution Georges Bailleau), appartenant à un ensemble décoratif réalisé par l'artiste, à Quibou; une tapisserie, issue de la collaboration entre l'architecte de l'église, Delaage, pour le carton et le maitre-lissier Jacques Plasse-Lecaisne à Saint-Hilaire-du-Harcouët. 


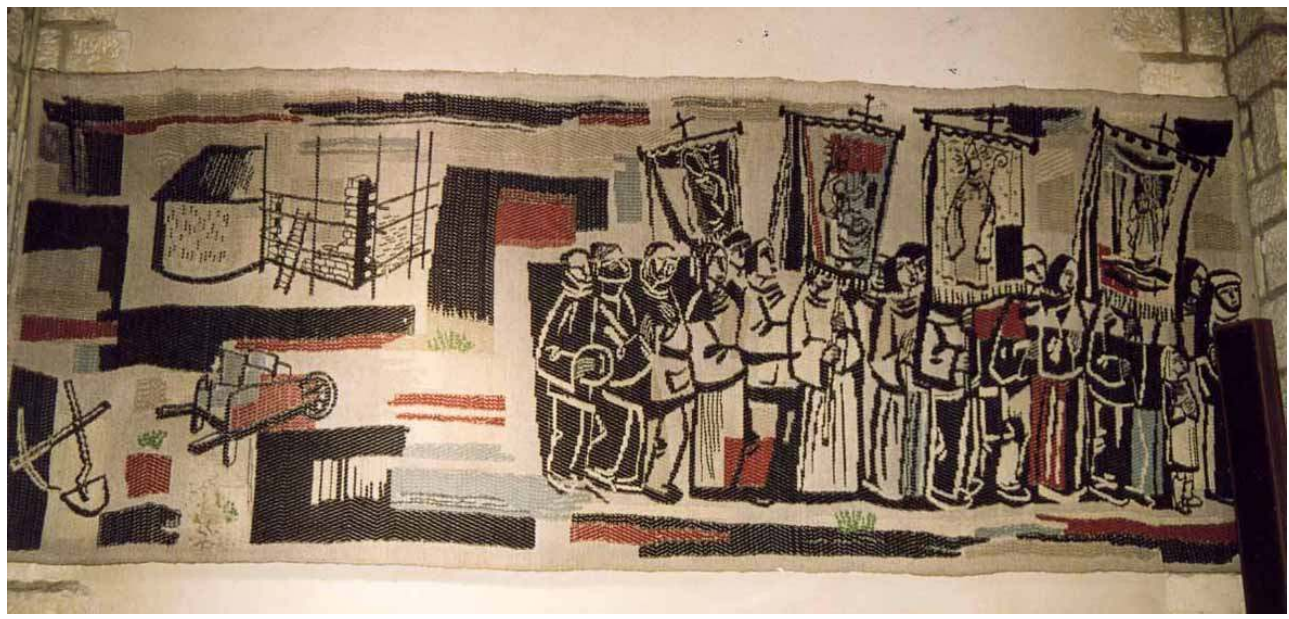

Tapisserie de Notre-Dame de la Salette par Chapuis et Plasse-Lecaisne, église du Mesnil-Véneron (c) Conseil général de la Manche/CAOA

Figure 21

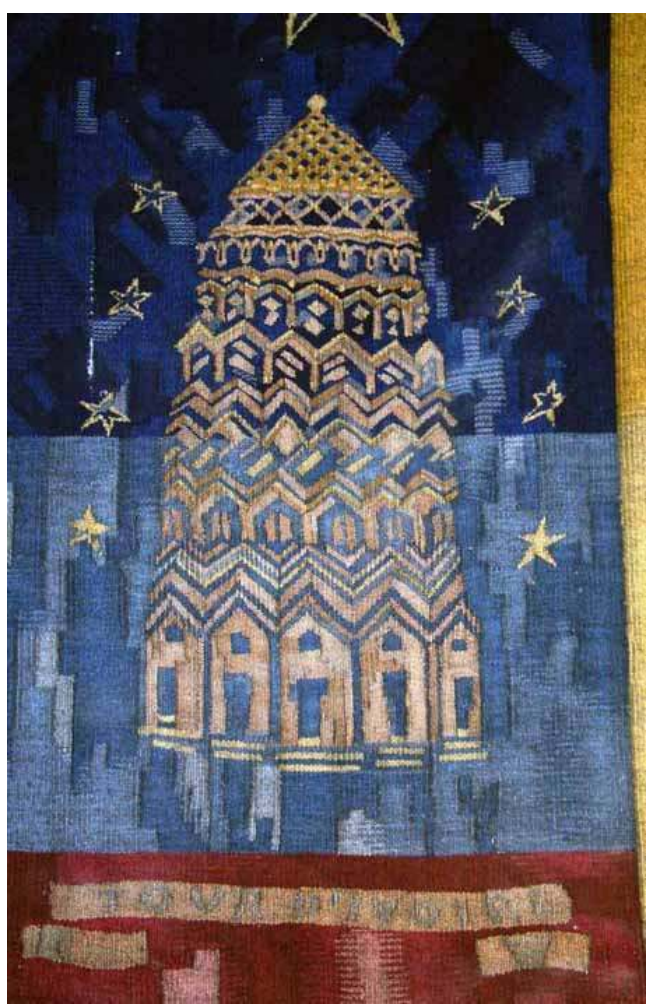

Tapisserie des symboles de la Vierge, par S. Flandrin-Latron et Ch. Giraud, église Notre-Dame de SaintLô

(C) Conseil général de la Manche/CAOA

Quatre chemins de croix ont été sélectionnés, représentatifs de différentes techniques (peinture à l'huile sur toile et sur bois, technique mixte, sculpture sur pierre): les chemins de croix d'Agneaux, Périers, Quibou et Martigny. Celui de Quibou (voir fig. $\mathbf{n}^{\circ} \mathbf{2}$ et fig. $\mathbf{n}^{\circ}$ 3), réalisé en 1955 par Helen Mai (pseudonyme de Jeanne Lartigue), très bien 
documenté grâce au rachat d'une partie du fonds d'atelier de l'artiste par un collectionneur, a été inscrit en raison de son intérêt artistique, sans tenir compte de son état de conservation alarmant (cf. ci-dessus). En comparaison avec celui de Notre-Dame de Saint-Lô (fig. $\mathbf{n}^{\circ} \mathbf{2 2}$ ), de Lucien Jeay, le chemin de croix de l'église de Périers (fig. $\mathbf{n}$ - 23), du même auteur, réalisé en 1961, en peinture à l'huile sur bois, a été jugé plus original et en même temps plus représentatif de l'œuvre de l'artiste que le précédent. Lucien Jeay, proposé par Yves-Marie Froidevaux, comme à Valognes et Saint-Lô, réalise à Périers son septième chemin de croix. Enfin, le chemin de croix de Martigny, œuvre peinte sur toile et marouflée sur bois, en 1958, du prêtre André Lecoutey (ancien membre des Ateliers d'Art Sacré aux côté de Georges Desvallières [1861-1950] et Maurice Denis [1870-1943]).

\section{Figure 22}

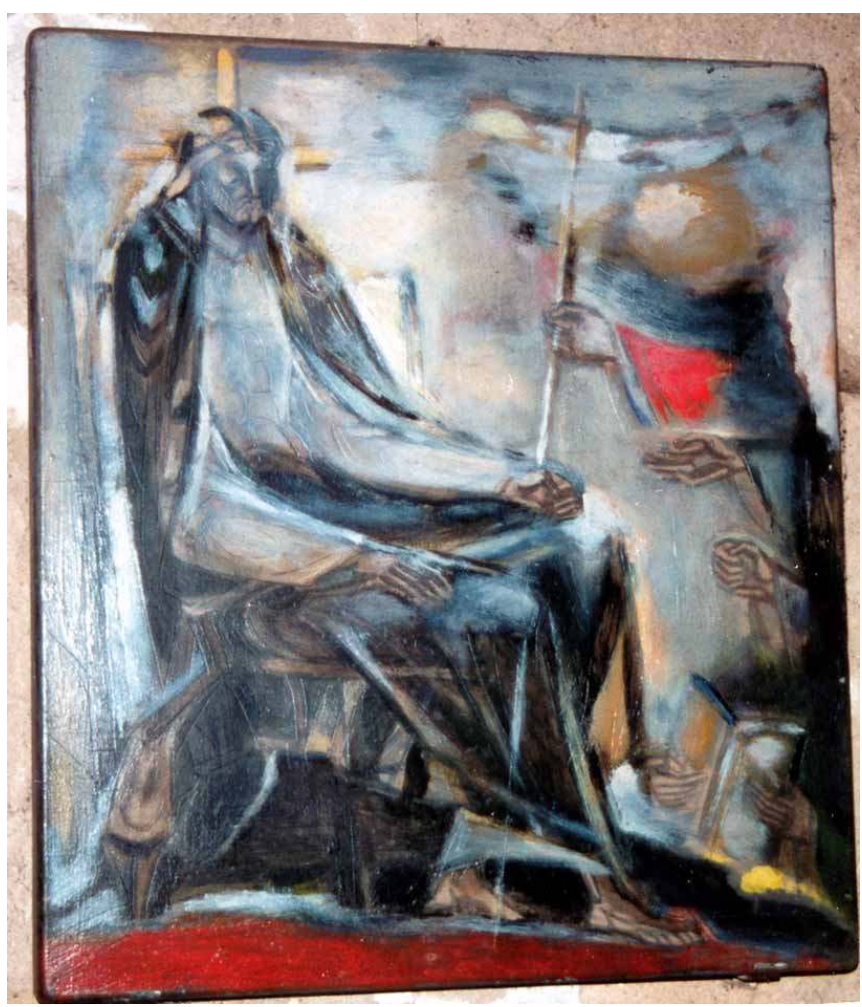

Chemin de croix de Périers

(c) Conseil général de la Manche/CAOA 
Figure 23

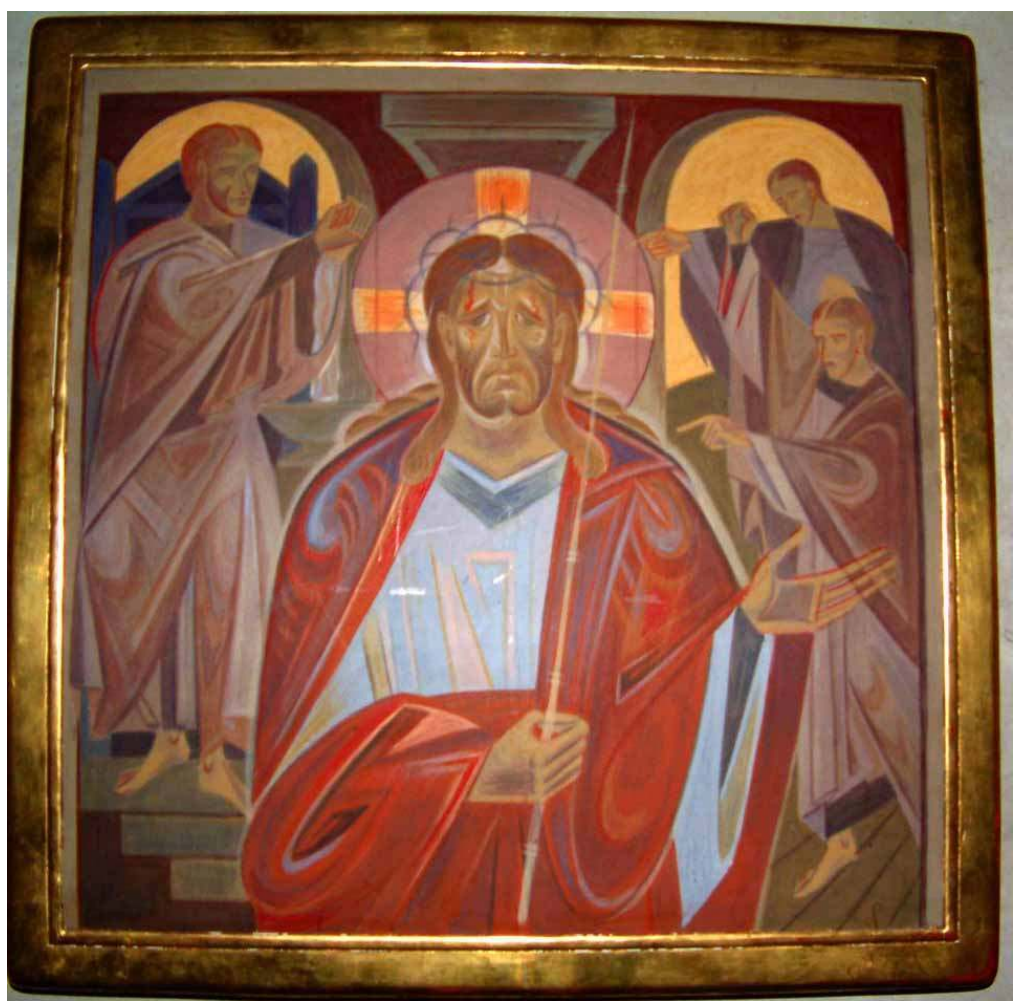

Chemin de croix de Notre-Dame de Saint-Lô

(C) Conseil général de la Manche/CAOA

Cinq éléments de mobilier liturgique (dont un ensemble) ont été sélectionnés. Le mobilier liturgique de l'église Saint-Malo de Valognes (fig. $\mathbf{n}^{\circ} \mathbf{2 4}, \mathbf{n}^{\circ} 25$ ) a été retenu et présenté comme un ensemble (autel et son retable, croix et chandeliers de chœur, ambon). 
Figure 24

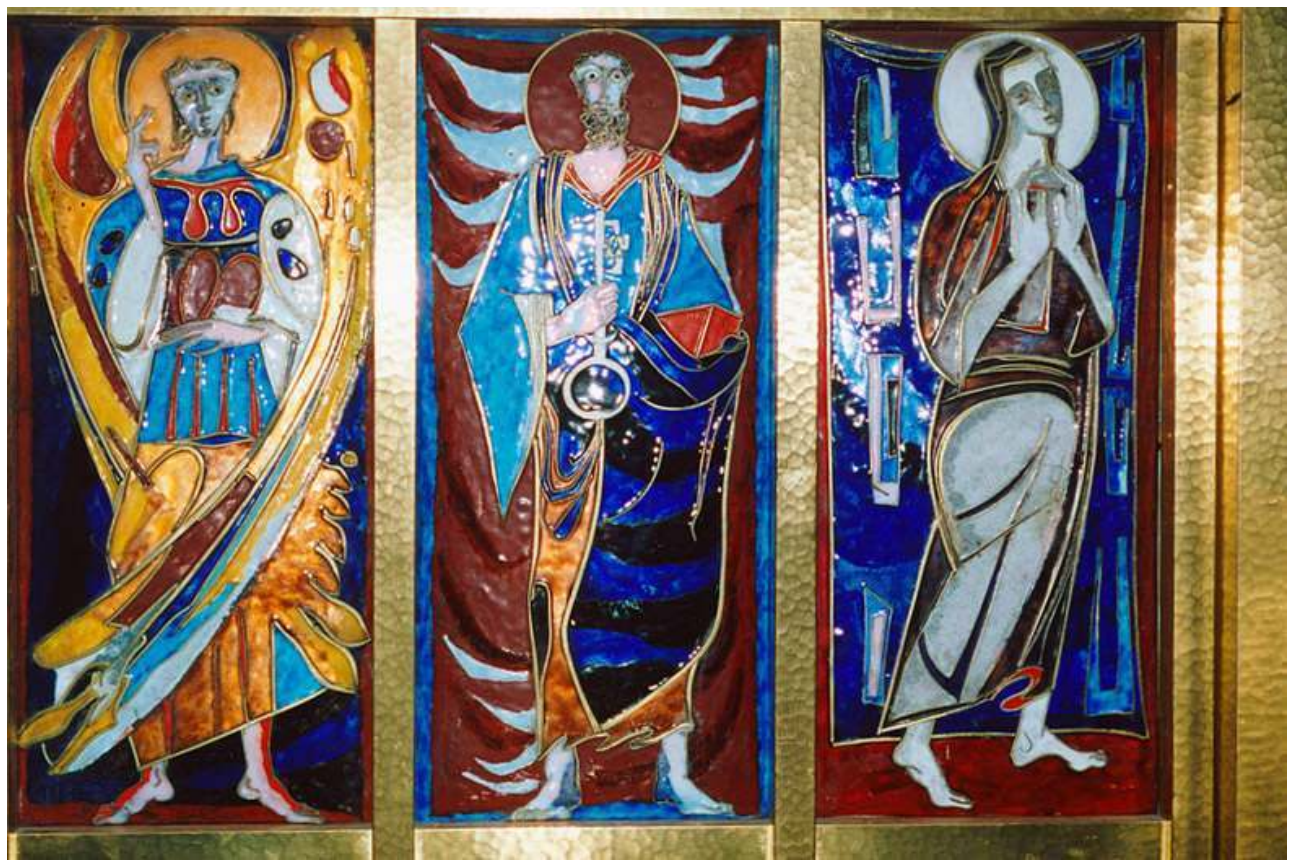

Détail de l'autel de Saint-Malo de Valognes (J. P. Froidevaux)

(c) Conseil général de la Manche/CAOA

\section{Figure 25}

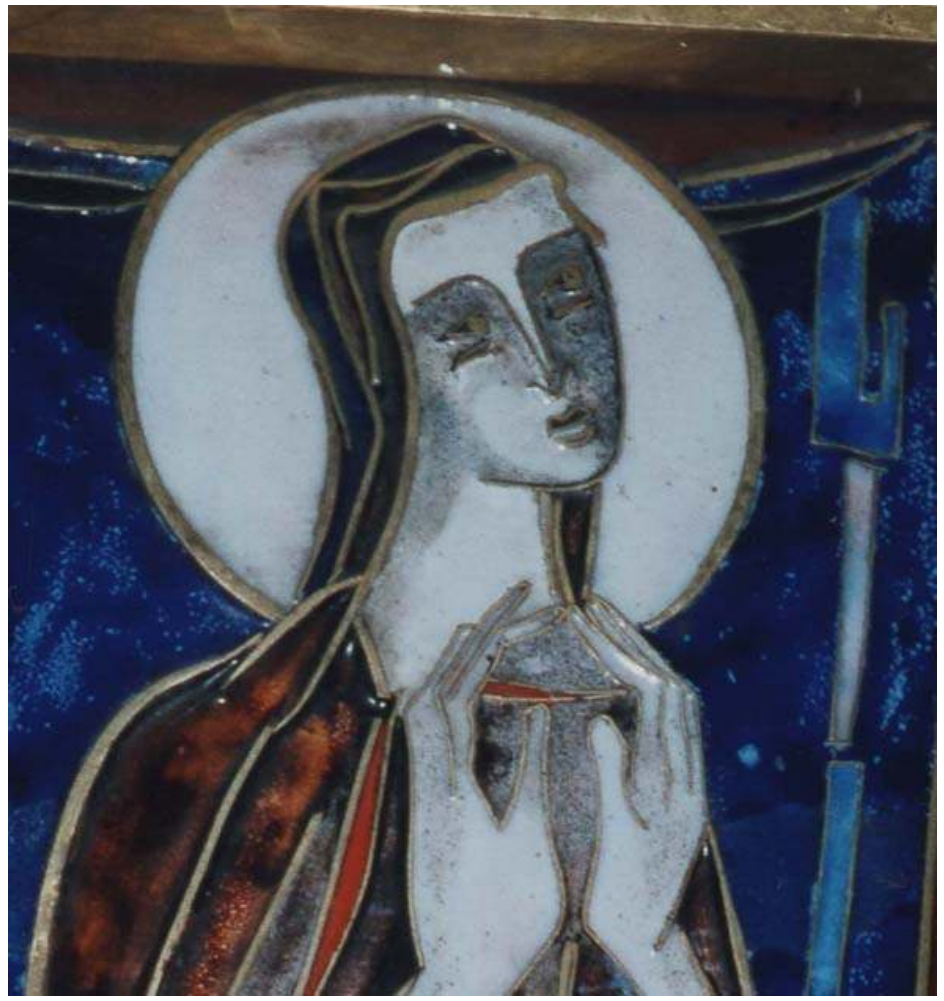

Détail des céramiques de l'autel de Saint-Malo de Valognes (J. P. Froidevaux) (c) Conseil général de la Manche/CAOA 

Yves-Marie Froidevaux (dessins du mobilier), Jean-Paul Froidevaux, son fils (cartons), la Maison Chéret (réalisation) et le sculpteur Jean Dambrin. Les autres pièces de mobilier sont un autel à Saint-Hilaire du Harcouët, fruit de la collaboration entre l'architecte Delaage et le sculpteur Rebuffet, ainsi qu'un tabernacle, à l'église de Périers. (fig. $\mathbf{n}^{\circ} \mathbf{2 6}$ )

Un seul ensemble d'orfèvrerie, une chapelle de Donat Thomasson (fig. $\mathbf{n}^{\circ} \mathbf{2 6}$ ) en argent martelé et jaspe roux, datant de 1947 s'est distingué des pièces inventoriées, œuvre originale de l'artiste du fait de la commande spécifique du prêtre de la paroisse, au sein d'une production marquée par son caractère répétitif.

Figure 26

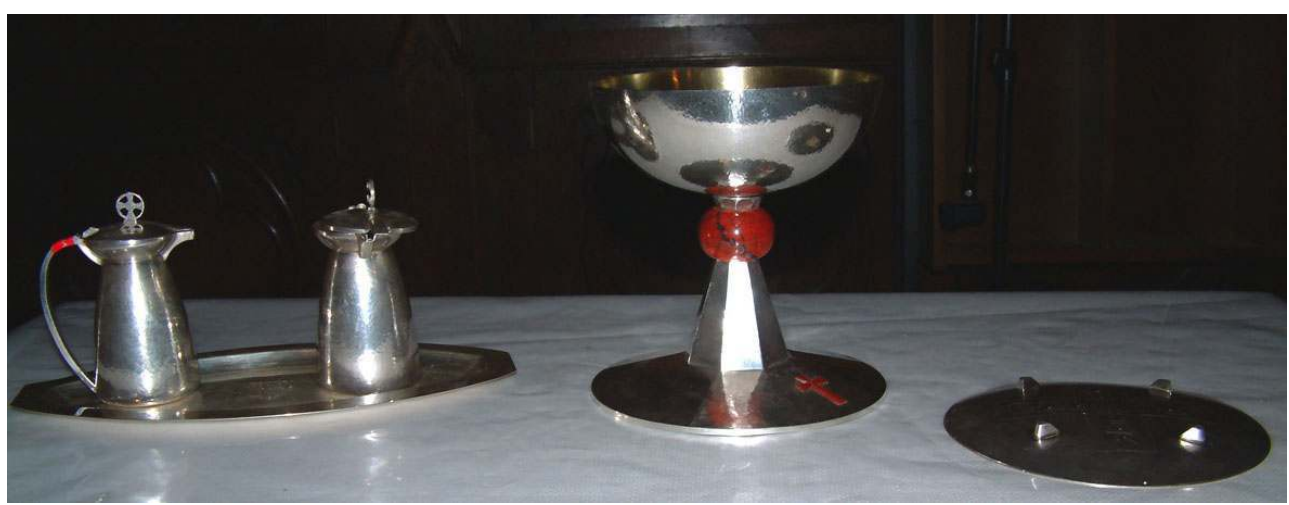

Chapelle de Donat Thomasson, paroisse de la Manche

(c) Conseil général de la Manche/CAOA

\section{Les enjeux de la protection}

Les questions concernant les rapports entre édifice et décor dans le processus de protection au titre des monuments historiques mettent en avant la nécessité de mener de manière concomitante les protections édifices et objets. 


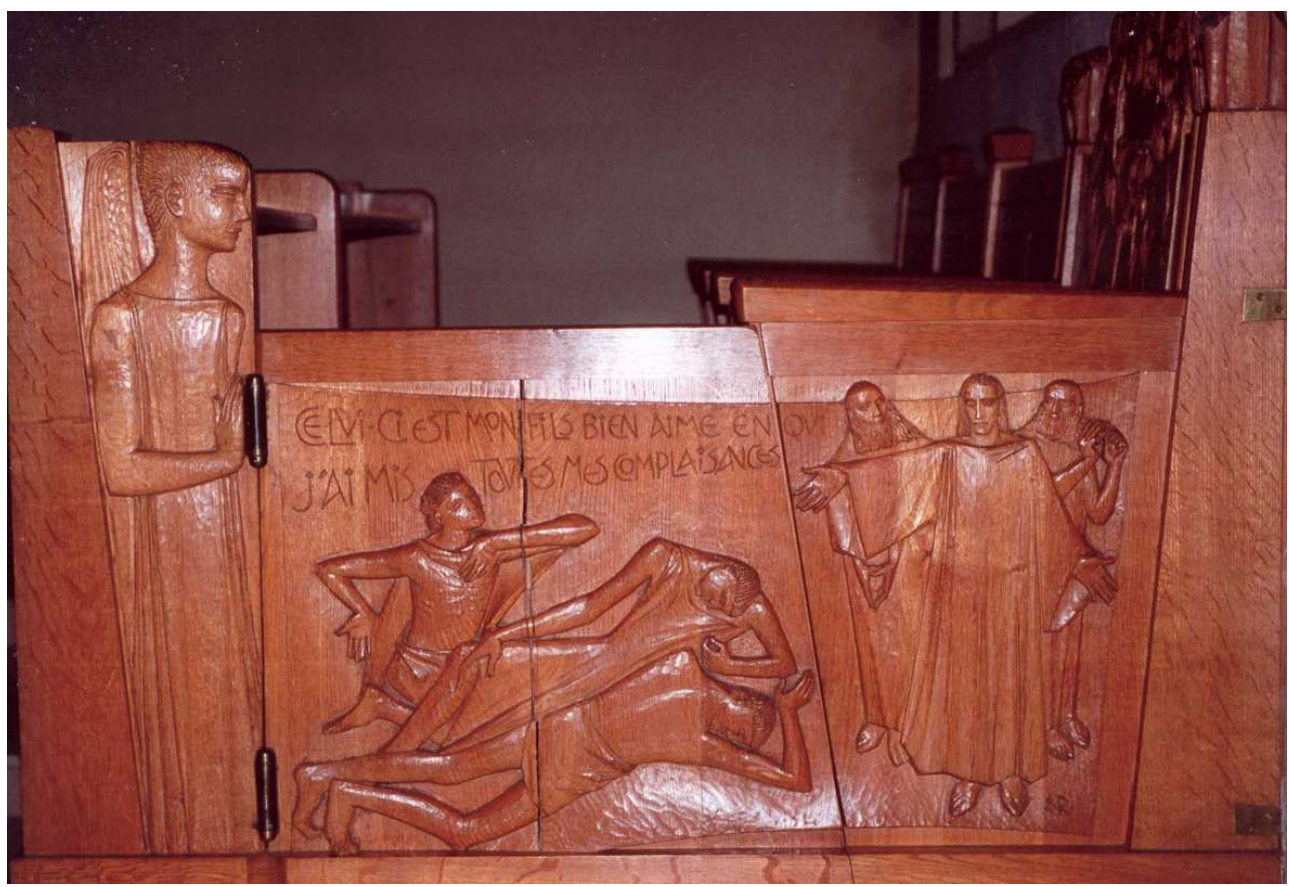

Stalles du Bon-Sauveur de Picauville (É. Rebuffet)

(C) Conseil général de la Manche/CAOA

\section{Figure 28}

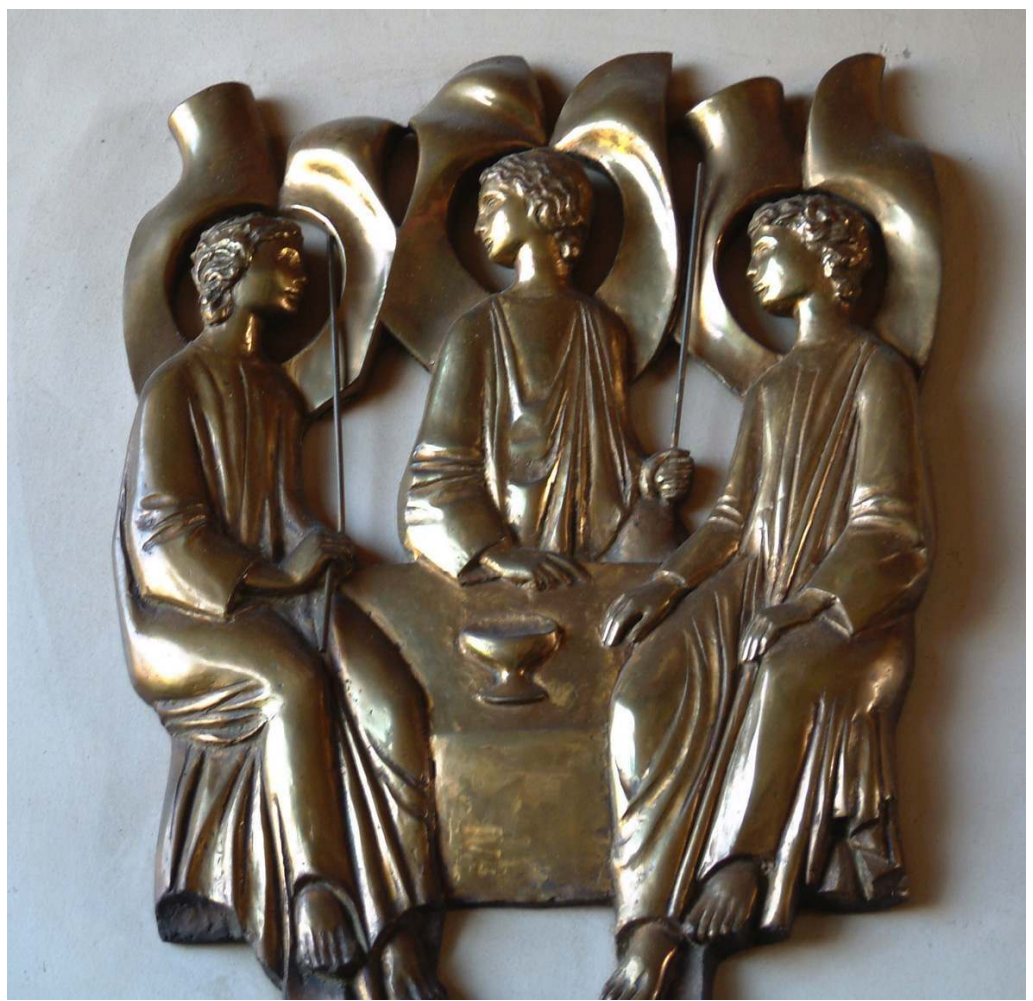

Bas-relief de I'hospitalité d'Abraham ou la Trinité du Bon-Sauveur de Picauville, par Étienne Rebuffet, modèle en bronze conservé dans le fonds d'atelier de l'artiste

(c) Conseil général de la Manche/CAOA 
La protection de l'ensemble du décor mobilier de la chapelle monastique du Bon-Sauveur de Picauville a été renvoyée en CRPS. La proposition ayant reçu un avis favorable, le décor figure expressément dans l'arrêté de protection de l'édifice sur lequel est annexée la liste précise des objets. Les œuvres sculptées sur bois sont d'Étienne Rebuffet: les stalles datant de 1953 (fig. $\mathbf{n}^{\circ} \mathbf{2 7}$ ) ornées de la Transfiguration et de l'Assomption et le bas-relief figurant la Trinité sous la forme de l'Hospitalité d'Abraham, surmontant le tabernacle, en bois doré à la feuille, datant de 1980, mais à situer dans la continuité de l'ensemble décoratif (fig. $\mathbf{n}^{\circ} \mathbf{2 8}$ ). Les éléments décoratifs en ferronnerie ont été réalisés par l'atelier parisien du décorateur Raymond Subes (1891-1970) : luminaires, grilles, portes, rampes d'escalier (fig. $\mathbf{n}^{\circ} \mathbf{2 9}, \mathbf{n}^{\circ} \mathbf{3 0}$ ).

Figure 29

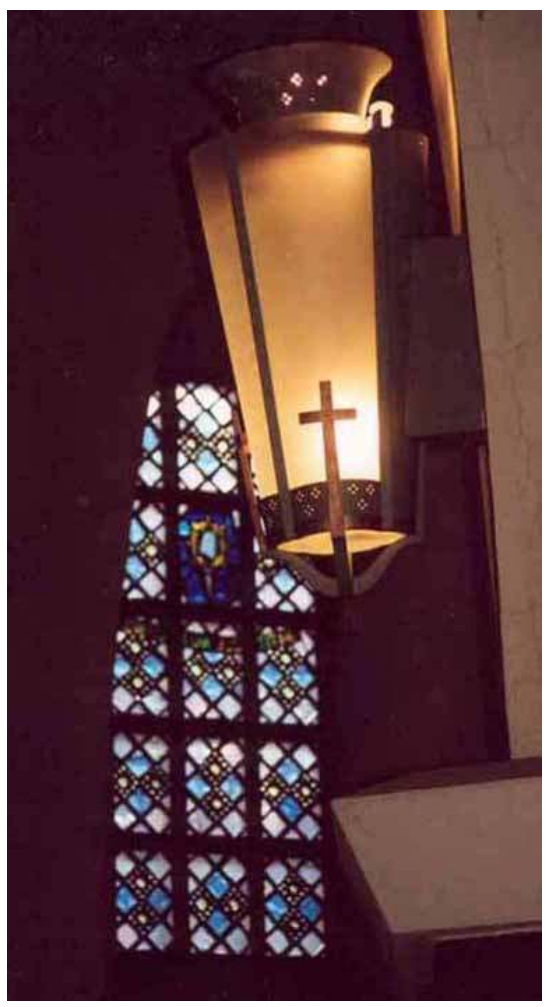

Portes de la chapelle du Bon-Sauveur de Picauville (R. Subes)

(C) Conseil général de la Manche/CAOA 


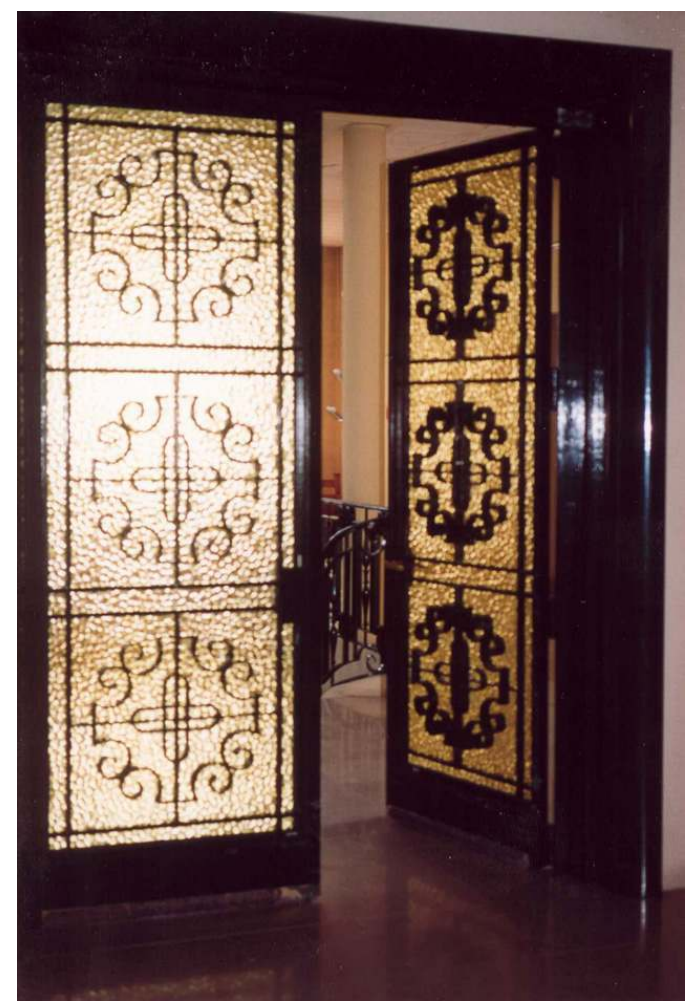

Luminaires de la chapelle du Bon-Sauveur de Picauville (R. Subes)

(C) Conseil général de la Manche/CAOA

31 La question s'est à nouveau posée concernant une tapisserie ornant l'église de Graignes, édifice également sélectionné pour la CRPS. La commission s'est prononcée en faveur de l'inscription en tant qu'objet, notamment du fait de l'urgence de la protection étant donné l'état alarmant de l'œuvre, mais aussi de l'issue incertaine de la protection édifice (pour laquelle la CRPS s'est prononcée favorablement par arrêté du 16 juin 2005). Enfin, le Chemin de croix de Saint-Hilaire-du-Harcouët, par Fanny Delaage, présenté pour sa fonction cultuelle, son intérêt iconographique et sa qualité artistique, réalisé à fresque sur les murs intérieurs d'un édifice, n'a pas été retenu pour la protection objet en raison notamment, du projet de présenter l'édifice pour l'inscription en CRPS. La présence de cette peinture, remarquée en CDOM, est venue appuyer le dossier de protection de l'édifice, mais, l'édifice n'ayant pas été protégé, le chemin de croix demeure non protégé (fig. $n^{\circ} 31, n^{\circ} 32$ ). 


\section{Figure 31}

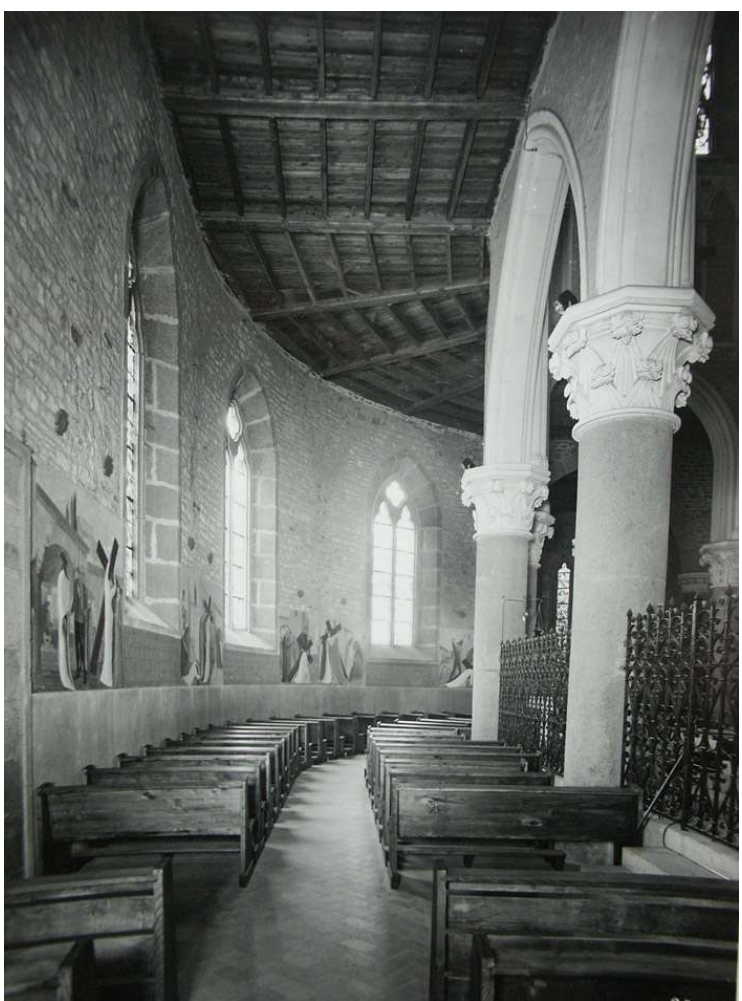

Déambulatoire de l'église de Saint-Hilaire-du-Harcouët (c) Conseil général de la Manche/CAOA 
Figure 32

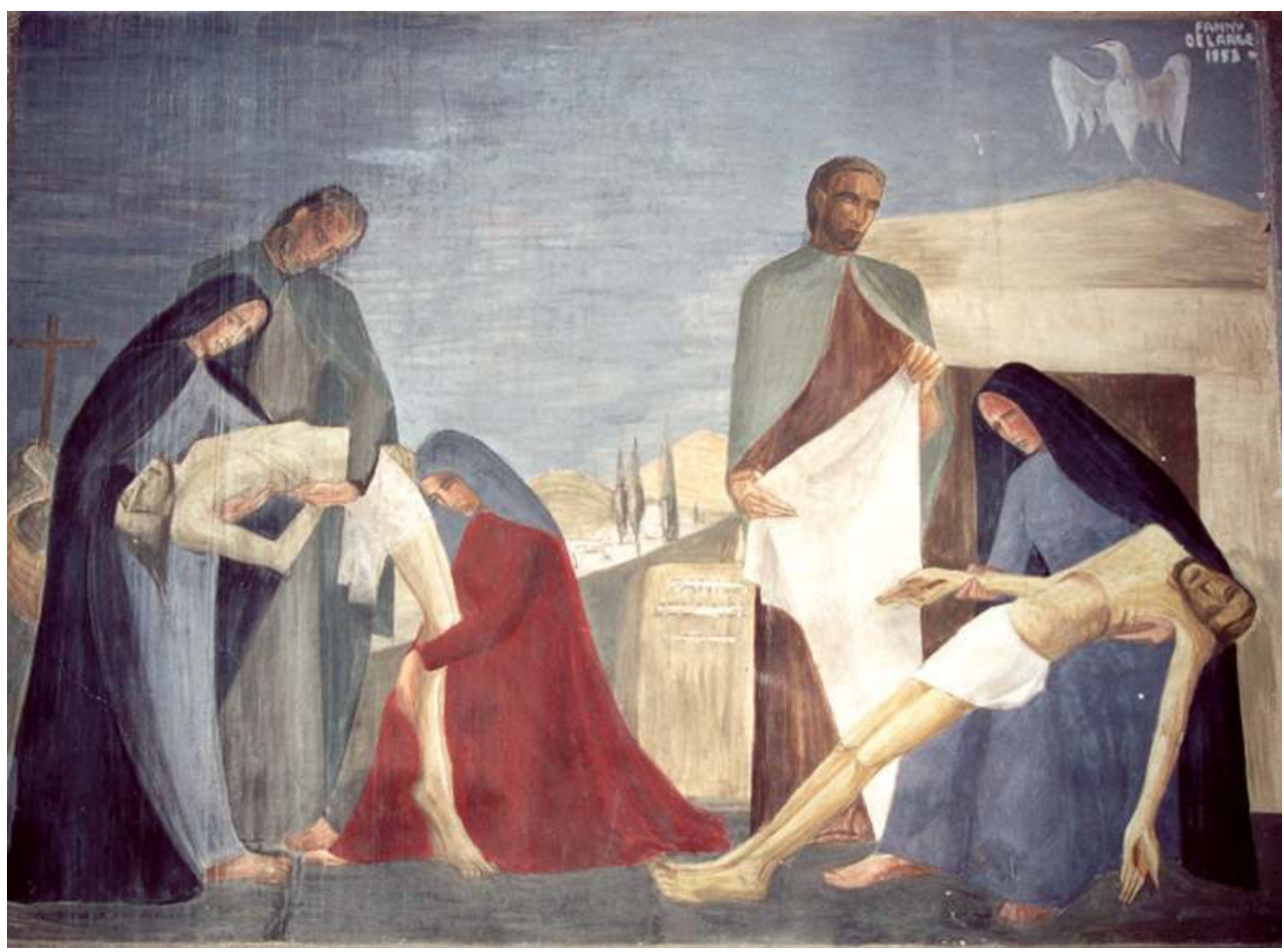

Deux dernières stations du chemin de croix de l'église de Saint-Hilaire-du-Harcouët (F. Delaage)

(c) Conseil général de la Manche/CAOA

32 Ces peintures étant, par ailleurs, œuvre d'une artiste vivante et restaurée récemment par cette dernière, à la demande de la commune propriétaire, en raison de leur mauvais état de conservation, la commission s'est montrée réservée sur l'opportunité de la protection. En effet, même s'il est vrai que d'un point de vue légal, rien ne s'oppose à la protection au titre des monuments historiques d'une telle œuvre, la patrimonialisation peut se révéler de fait, délicate, les travaux sur l'œuvre étant soumis au droit moral de l'auteur. 
Figure 33

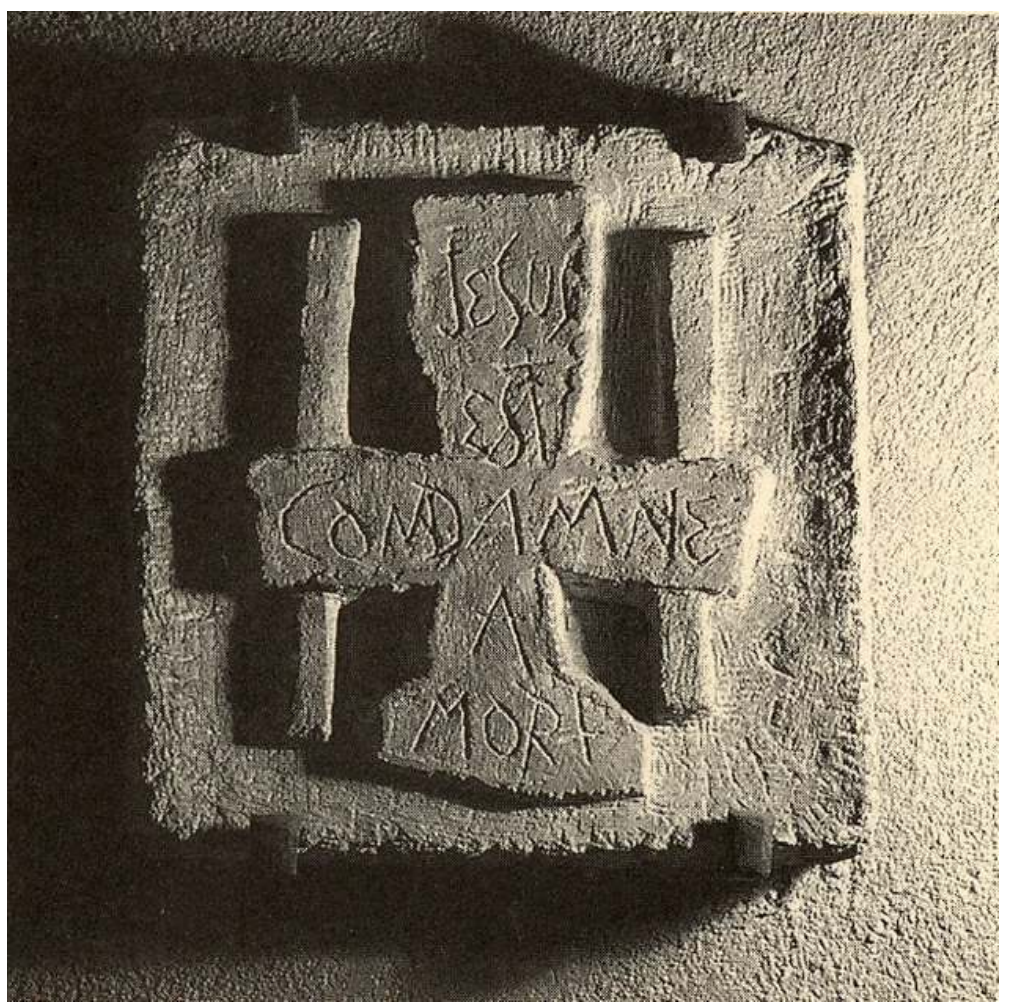

Première station du chemin de croix de l'église d'Agneaux (L. Zack). Photo extraite de Bellego, Pierre. Chemin de croix, 2000

Phot. Bertrand Lebas. (c) APB Association des Amis de Pierre Bellégo

Autre question soulevée en CDOM, celle des éventualités de classement, concernant plusieurs objets. En effet, si les critères ont pu être affinés au niveau de l'inscription, grâce à la connaissance du corpus bas-normand spécialement concerné par la Reconstruction, la sélection d'objets en vue du classement restait encore très relative. De ce fait, les souhaits de la CDOM sur ce sujet sont restés sans suite. Le chemin de croix d'Agneaux, fruit de la collaboration entre Léon Zack et Maxime Adam-Tessier, par exemple, présente un intérêt qui dépasse le simple plan local, tant par sa forme, très « moderne » qui privilégie un retour à la taille directe, que par le traitement abstrait du sujet, ou la force d'évocation qu'il dégage ${ }^{6}\left(\right.$ fig. $\mathbf{n}^{\circ} 33$ ). Sur un fond simplement dégrossi dans le calcaire, ou gravé de petites hachures, des croix obliques ou verticales se détachent en creux ou en relief, accompagnées d'inscriptions et de symboles se rapportant à la Passion (clous, suaire, éclair,...). La croix se substitue au Christ, dont la figure est absente, et fait corps avec elle (les obliques faisant référence aux chutes du Christ). Les oppositions de formes, les contrastes dans le traitement du matériau (griffures et gravures se heurtant aux zones polies), symbolisent les étapes du chemin de croix. Par comparaison, le chemin de croix de Carsac, œuvre du même auteur (aidé d'Irène, sa fille) et semblable à celui d'Agneaux mais réalisé en terre-cuite, a été classé en $2005^{7}$. À ce jour, douze chemins de croix datant du XXe siècle ont bénéficié de cette mesure de protection, dont trois sont de Maurice Denis. Onze datent donc de la première moitié du XXe siècle ${ }^{89}$. 


\section{Conclusion}

Figure 34

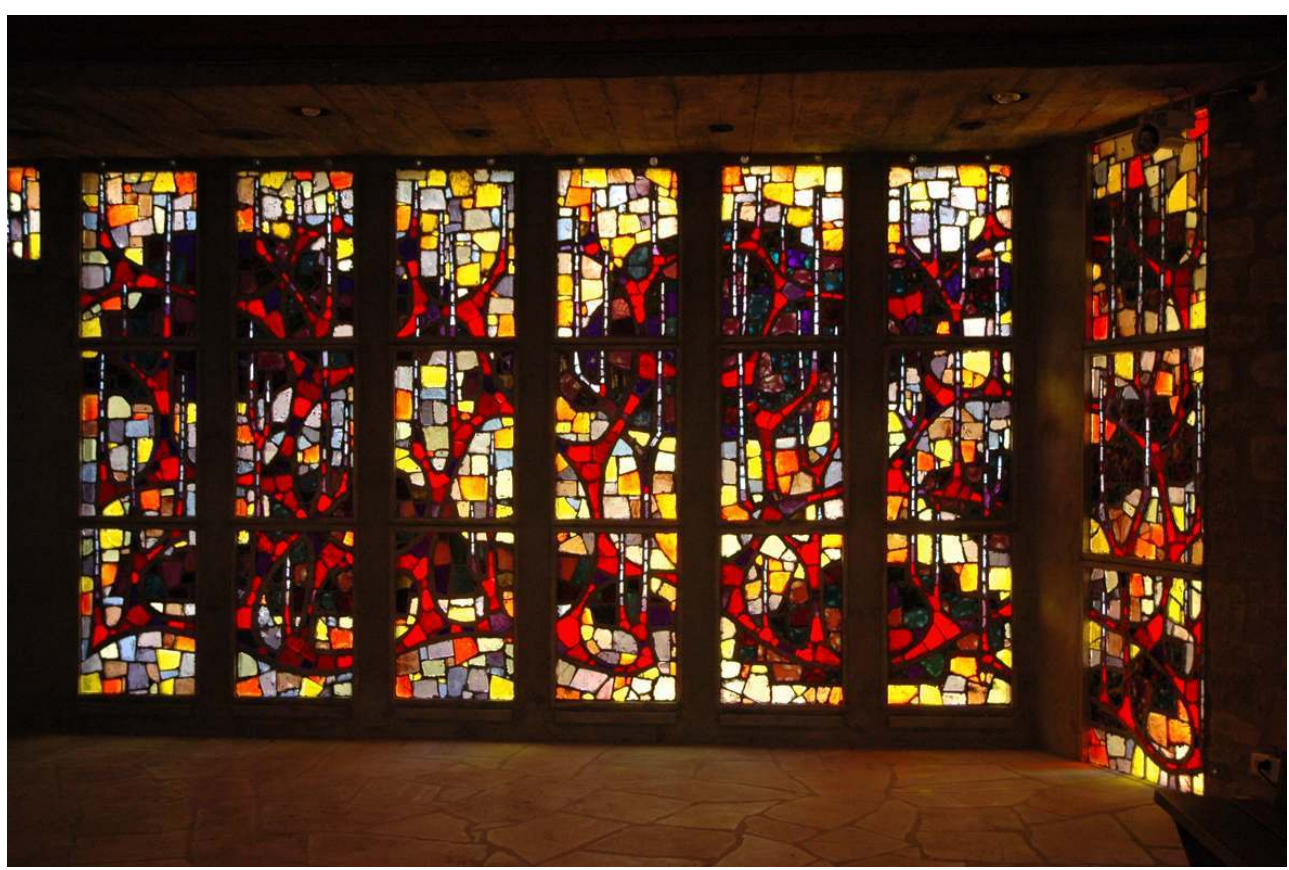

L'arbre de Jessé, dalles de verre de l'église d'Urville-Nacqueville (H. Martin-Granel)

(c) Conseil général de la Manche/CAOA

Les protections ont permis d'alerter les municipalités sur l'intérêt de leur patrimoine contemporain et d'entreprendre des restaurations. Elles ont parfois servi d'élément déclencheur pour des valorisations in situ : réfection d'enduits (à l'église de Pont-Hébert), installation d'éclairages pour mettre en valeur les objets, commande de mobilier liturgique, en liaison avec certains architectes, artistes ou leurs familles (la maison Chéret pour la commande de mobilier à Notre-Dame de Saint-Lô, Jacques Prioleau et Irène Zack pour la réfection des enduits, l'éclairage et le nettoyage du chemin de croix à l'église d'Agneaux, la famille Martin-Granel ${ }^{10}$ pour la restauration des dalles de verre à UrvilleNacqueville). (fig. $\mathbf{n}^{\circ}$ 34)

INP

Figure 35

\section{in Institut national du patrimoine}

Communication présentée lors du séminaire LE PATRIMOINE RELIgIEUX DES XIX ${ }^{\mathbf{E}}$ ET $\mathbf{X X}^{\mathbf{E}}$ SIÈCLE qui s'est tenu du 9 au 11 juin 2008 à l'Institut national du patrimoine, avec la participation de la direction de l'Architecture et du Patrimoine 


\section{NOTES}

1. Ce texte reprend, sous une forme un peu différente, une communication faite au colloque tenu à Lyon en 2006 et paru, sous le titre «Le mobilier religieux reconstruction (1945-1960) de la Manche ", dans Regards sur l'objet du XX ${ }^{\mathrm{e}}$ siècle, Actes Sud, 2007, p. 153-162. Ce travail, auquel j'associe Josiane Pagnon et Clara Lemoussu, est le fruit d'une collaboration étroite au sein de la conservation. Je remercie Alain Nafilyan pour son aide et le prêt de clichés. Fiches détaillées des objets et dépouillements d'archives consultables (au fur et à mesure des mises à jour) sur : http:// objetart.cg50.fr.

2. D'après une enquête statistique d'édifices détruits, réalisée en octobre 1944 auprès des prêtres, à l'initiative de $\mathrm{M}^{\mathrm{gr}}$ Aubry (archives diocésaines de Coutances).

3. Voir dans ce numéro l'article d'Alain Nafilyan : La reconstruction des édifices religieux en Basse-Normandie après la Seconde Guerre mondiale.

4. Plaquettes Églises de la Reconstruction, Conseil général de la Manche (conservation des antiquités et objets d'art), 2004.

5. Cet inventaire qui concerne désormais les 160 églises, et qui pourra s'étendre au patrimoine civil, doit aboutir à une publication et une exposition. La couverture du patrimoine vitré est déjà bien avancée et mise en ligne au fur et à mesure sur le site internet : http://objetart.cg50.fr.

6. L'œuvre a été choisie pour servir d'illustration au Chemin de croix de Pierre Bellego, édité en 2000, accompagné de l'enregistrement du texte lu à Saint-Séverin par Michaël Lonsdale et mis en musique par François Espinasse, organiste.

7. Voir dans la base Palissy : notice PM24000694.

8. Voir à ce sujet: Bouillet, Jean-Roch. «La commande publique contemporaine : l'exemple des chemins de croix en Provence-Alpes-Côte d'Azur » dans Regards sur l'objet du $\mathbf{X X}^{\mathrm{e}}$ siècle, Actes Sud, 2007, p. 133-142.

9. Voir également dans le numéro 12 de la revue, l'article d'Alain Nafilyan : Le monastère des bénédictines à Couvrechef, Caen (Calvados); et celui de Véronique David : Castro et le défi du vitrail.

10. Pour avoir accès à l'enquête orale de la famille Martin-Granel, réalisée par l'équipe de recherche sur le vitrail au Centre André Chastel, Institut national d'histoire de l'art, il suffit d'en faire la demande par messagerie à Beatrice.Coquet@paris-sorbonne.fr

\section{RÉSUMÉS}

Plus de trois cents églises de la Manche ont été détruites ou gravement endommagées en 1944. La Reconstruction, étalée sur une trentaine d'années, est devenue une composante du patrimoine départemental. La Conservation des antiquités et objets d'art a entrepris un inventaire de ce patrimoine qui concerne cent soixante édifices et environ cent trente objets, auxquels s'ajoutent les ensembles de vitraux. Dans un premier temps, trente édifices ont été inventoriés, parallèlement à l'étude menée sur les édifices par le service des Monuments historiques de Basse- 
Normandie. Ces deux enquêtes ont été suivies de mesures de protection concernant quarante objets ou ensembles mobiliers conservés dans les églises de la Manche. Les critères généraux de protection au titre des monuments historiques ont été débattus, affinés et précisés lors de deux commissions départementales des objets mobiliers thématiques.

In 1944 more than three hundred churches in the Manche department were destroyed or badly damaged. The reconstruction of these churches took over thirty years, and these reconstructed churches are now recognised as an integral part of the department's heritage. The services of the Conservation des antiquités et objets d'art has undertaken an inventory of these churches, focussed on 160 buildings and about 130 religious artefacts, along with a collection of stained glass. To begin with, the contents of thirty churches were surveyed in an operation running alongside an inventory of religious buildings carried out by the Historic monuments service of the Basse-Normandie region. These two surveys were followed by measures of statutory protection for forty objects or furnishing ensembles conserved in the department's churches. There was considerable debate as to the criteria appropriate for the protection of these objects, and two meetings of the departmental commission on the protection of the movable heritage were able to refine these criteria and render them more pertinent.

\section{INDEX}

Mots-clés : Henri Martin-Granel, église Saint-Sébastien du Plessis, abbatiale de Lessay, Étienne Rebuffet, École de Paris, Plessis-Lastelle, Maurice Denis, Famille Martin-Granel, Église d'UrvilleNacqueville, Patrimoine religieux du XXe siècle, Patrimoine religieux du 20e siècle, Patrimoine religieux de la Manche, Patrimoine religieux Basse-Normandie, Mobilier religieux de la Manche, Mobilier religieux du XXe siècle, Mobilier religieux de la Reconstruction, Reconstruction Manche, Chemin de croix, Sculpture religieuse du XXe siècle, Statuaire religieuse du XXe siècle, Objets d'art du XXe siècle, Mobilier liturgique du XXe siècle, CRPS Basse-Normandie, André Lecoutey, Maison Chéret, Jean Dambrin, Donat Thomasson, chapelle monastique du Bon-Sauveur de Picauville, Raymond Subes, Fanny Delaage, Église de Carsac, Irène Zack, Église de Pont-Hébert, Conservation des antiquités et objets d'art de la Manche, CAOA Manche, Église de Laulne, Église de Saint-Jean-des-Baisants, Églises de la Reconstruction, Jeanne Lartigue, église de Graignes, François Chapuis, église Notre-Dame de Saint-Lô, Jean Bernard, églises de la Manche, église SaintJean-Bastiste de Lastelle, église d'Agneaux, Maurice Rocher, Ghiglione, Georges Pouleaud, église Saint-Malo de Valognes, Yves-Marie Froidevaux, Simone Flandrin-Latron, Max Ingrand, JeanPaul Froidevaux, Lucien Jeay, Jean Dambrin, église de La Haye-du-Puits, CDOM Manche, Jacques Prioleau, Léon Zack, Maxime Adam-Tessier, Jean Lambert-Rucki, Philippe Kaëppelin, Label Patrimoine XXe, Église de La Chapelle-Enjuger, Église du Mesnil-Véneron, Jacques PlasseLecaisne, Charles Giraud, Georges Bailleau, Delaage, Église de Saint-Hilaire-du-Harcouët, Église de Périers, Église de Martigny, église de Quibou, Helen Mai

\section{AUTEUR}

\section{ÉLISABETH MARIE}

Conservatrice déléguée des objets d'art, conseil général de la Manche. Elisabeth.marie@cg50.fr 